\title{
Tasmania Hemp (Cannabis) Fiber and Seed Cultivar Field Trials—2018-2019
}

\author{
Robert C. Clarke \\ BioAgronomics Group Consultants, Los Angeles, California, USA.
}

How to cite this paper: Robert C. Clarke. (2020) Tasmania Hemp (Cannabis) Fiber and Seed Cultivar Field Trials-2018-2019. International Journal of the Science of Food and Agriculture, 4(4), 470-481.

DOI: $10.26855 /$ ijfsa.2020.12.016

Received: November 8, 2020

Accepted: November 30, 2020

Published: December 22, 2020

*Corresponding author: Robert C. Clarke, BioAgronomics Group Consultants Los Angeles, California, USA. Email: rob@bioagronomics.com

\begin{abstract}
Tasmania has a temperate climate well-suited for hemp production, is located nearer to the south pole than other regions of Australia, and therefore presents an important location for hemp variety trials. The primary aim of this research is to evaluate the performance of hemp (Cannabis) fiber and seed cultivars from various geographical origins and latitudes when grown in southern Tasmania. Seventeen registered hemp cultivars were sown on three dates in large replicate trial blocks to allow combine harvesting and collection of accurate yield data. Results are discussed with respect to site selection, crop performance, sowing dates, sowing rates, crop development, pest infestation, THC levels, seed yields, crop management and cultivar selection. Canadian cultivars performed well for grain seed production with the highest yields and their short crop height accommodates mechanical harvesting. French cultivars yielded much less seed than Canadian cultivars and grew to heights nearly beyond the reach of a standard grain header. Chinese cultivars flowered too late to produce viable seeds and are not suitable for hemp seed grain production in Tasmania, although their vigorous growth and late flowering makes them good candidates for biomass production. THC levels in the sampled Chinese cultivars were too high for seed production, but those that do not flower at Tasmanian latitudes are suitable for fiber and biomass production.
\end{abstract}

\section{Keywords}

Tasmania, Cannabis, hemp, seed, trials

\section{Introduction}

Results of three hemp cultivar trials from Australia have been published (Table 1). During the 1999-2000 season, trials were sown on one site at Murphys Creek near Toowoomba in Queensland $\left(27.5^{\circ} \mathrm{S}\right)$ with the intention of identifying high stalk/biomass yielding varieties. Three Australian selections of Asian origin, French fiber and seed cultivar 'Futura 77', Hungarian fiber cultivar 'Kompolti' and Finnish hempseed cultivar 'FIN 314' (AKA 'Finola') were trialed at four staggered sowing dates every 15 days ranging from the end of October through mid-December. Results indicated that the latitude of origin of each variety was the determining factor in its flowering time and that later flowering was related to higher stalk yield. Asian selections originating from semi-tropical latitudes flowered later and produced more biomass than European cultivars from temperate latitudes [1].

In 2016-2017, trials were grown at Forthside near Launceston in the north of Tasmania $\left(41.2^{\circ} \mathrm{S}\right)$ to assess hemp cultivars for grain seed production. Twelve Australian breeder's selections and three hemp seed cultivars, 'CFX' and 'CRS-1' from Canada and Finnish 'Finola', were compared in three sowings ranging from late November through early January. The breeder's selections from landrace introductions generally yielded more seed than the Canadian and Finnish cultivars which is promising for the future of breeding Australian hemp seed cultivars [2].

Trials were conducted during the 2017-2018 season at Loxton (34.4 $\left.{ }^{\circ} \mathrm{S}\right)$ and Kybybolite (36.9 ${ }^{\circ}$ ) in South Australia. 
Two Australian breeder's selections, biomass cultivar 'Frog 1' from Australia and fiber and seed cultivars 'Ferimon 12' from France and 'Han-NE' from China were trialed in five sowings from late October through mid-January [3]. Seed yields were yet to be published when the 2018-2019 Bothwell field trial began.

Latitude determines daylength and daylength controls the flowering response in Cannabis plants. Hemp cultivars flower as the day length decreases in late summer and early autumn and when grown at their latitude of origin they ripen in early autumn before winter arrives. If hemp cultivars are grown at another latitude they will respond differently. When grown closer to the equator they flower prematurely, the plants are shorter, and the seeds may not ripen evenly. When grown closer to the poles they flower later than at their native latitude, plants grow taller and their seeds may not mature before the frost.

Table 1. Years, locations, latitudes, maximum daylengths and climate types of previous Australian hemp variety trials

\begin{tabular}{lllll}
\hline Years & Locations & Latitudes & $\begin{array}{c}\text { Daylengths } \\
\text { (max. hours) }\end{array}$ & Climate Types \\
\hline $1999-2000$ & Murphys Creek, Queensland & $27.5^{\circ} \mathrm{S}$ & 14.1 & Warm temperate \\
$2017-2018$ & Loxton, South Australia & $34.4^{\circ} \mathrm{S}$ & 14.3 & Warm temperate \\
$2017-2018$ & Kybybolite, South Australia & $36.9^{\circ} \mathrm{S}$ & 14.5 & Warm temperate \\
$2017-2018$ & Forthside, Tasmania & $41.2^{\circ} \mathrm{S}$ & 15.1 & Cool temperate \\
$2018-2019$ & Bothwell, Tasmania & $42.4^{\circ} \mathrm{S}$ & 15.2 & Cool temperate \\
\hline
\end{tabular}

\section{Materials and Methods}

\subsection{Cultivars}

The 2018-2019 Tasmanian hemp cultivar field trials included 17 cultivars and of these only 'CRS-1' from Canada and 'Han-NE' from China were sown in previous Australian cultivar trials. The Canadian seed cultivars and the Chinese fiber and seed cultivars are dioecious with male and female flowers borne on separate plants, and the French fiber and seed cultivars are monoecious with both male and female flowers borne on the same plant. Germination percentages for the Canadian cultivars ranged from 84 to 97 percent, the French cultivars ranged from 89 to 95 percent and the Chinese cultivars ranged from 33 to 97 percent.

Based on the latitudes of origin of the hemp cultivars to be trialed we made several predictions of general outcomes. The Canadian seed cultivars ('CFX-1', 'CRS-1', 'Katani' and 'Picolo') originate from approximately $52.0^{\circ} \mathrm{N}$ latitude and the French fiber and seed cultivars ('Felina 32', 'Ferimon', 'Futura 75' and 'Santhica 70') originate from approximately $48.0^{\circ} \mathrm{N}$ latitude. The Chinese fiber and seed cultivars purportedly originate from higher ('Han FN H', 'Han FN Q' and 'Han Cold') and middle ('Han NE' and 'Han NW') northern temperate latitudes as well as tropical and sub-tropical latitudes ('Bama', 'Puma-3', 'Yuma' and 'Si-1'). Bothwell is located at $42.4^{\circ} \mathrm{S}$ and the Canadian and French cultivars were expected to flower earlier than at their latitude of origin while the Chinese cultivars should flower later in the season.

\subsection{Determination of seed viability}

In order to sow the correct amount of seed, aiming at high stand density sufficient to produce a closed canopy and suppress weed establishment, seed viability must be taken into account (Table 2). The viability of imported seeds was determined by placing 100 seeds of each cultivar in warm moist paper towels for several days and counting those that germinated to calculate the germination percentage. The vigor of sprout growth was also noted. Once the germination percentage was determined it was factored in when determining the amount of seed to be sown per square meter. Target sowing densities were 150 seeds per square meter for more viable seeds (81 to 97 percent germination) and 200 seeds per square meter for less viable seeds (35 to 65 percent germination).

\subsection{Field preparation}

Trial plots were prepared employing standard broad-acre field management strategies for soil preparation, nutrient supplementation and weed control. The E trial site soil was medium sandy loam, slightly acidic (pH 6.2) with medium EC (353 ppm), low calcium (1,231 ppm) content and a low Ca:Mg ratio (3.59:1). The R trial site soil was slightly acidic (pH 6.3) clay-rich sandy loam, with a higher EC (640 ppm) level and calcium content (1,714 ppm) and a lower 
Ca:Mg ratio (2.97:1).

Table 2. Cultivars, origins, sexual types, seed weights, germination percentages, germination rates and seedling vigor (2018)

\begin{tabular}{|c|c|c|c|c|c|c|c|c|c|c|}
\hline \multirow[t]{2}{*}{ Cultivar } & \multirow[t]{2}{*}{ Origin } & \multirow[t]{2}{*}{ Sexual Type } & \multirow{2}{*}{$\begin{array}{l}1000 \text { Seed } \\
\text { Weight }(\mathrm{gr})\end{array}$} & \multicolumn{5}{|c|}{ Germination Percentage } & \multirow{2}{*}{$\begin{array}{l}\text { Germination } \\
\text { Rate }\end{array}$} & \multirow{2}{*}{$\begin{array}{l}\text { Seedling } \\
\text { Vigor }\end{array}$} \\
\hline & & & & $\begin{array}{c}48 \\
\text { hours }\end{array}$ & $\begin{array}{c}72 \\
\text { hours }\end{array}$ & $\begin{array}{c}96 \\
\text { hours }\end{array}$ & $\begin{array}{c}120 \\
\text { hours }\end{array}$ & $\begin{array}{c}144 \\
\text { hours }\end{array}$ & & \\
\hline 'CFX-1' & Canada & Dioecious & 17.0 & 87 & 88 & 89 & 90 & 90 & Fast & High \\
\hline 'CRS-1' & Canada & Dioecious & 17.8 & 91 & 93 & 94 & 94 & 94 & Fast & High \\
\hline 'Katani' & Canada & Dioecious & 16.2 & 92 & 95 & 96 & 97 & 97 & Fast & High \\
\hline 'Picolo' & Canada & Dioecious & 16.5 & 80 & 81 & 83 & 84 & 84 & Medium & High \\
\hline 'Felina 32' & France & Monoecious & 17.8 & 72 & 90 & 92 & 93 & 95 & Medium & High \\
\hline 'Ferimon' & France & Monoecious & 17.1 & 78 & 91 & 92 & 94 & 94 & Medium & High \\
\hline 'Futura 75’ & France & Monoecious & 18.8 & 69 & 80 & 83 & 88 & 89 & Medium & High \\
\hline 'Santhica 70' & France & Monoecious & 18.1 & 70 & 87 & 92 & 93 & 94 & Medium & High \\
\hline 'Han FN H’ & China & Dioecious & 22.2 & 28 & 48 & 55 & 58 & 62 & Slow & Low \\
\hline 'Han FN Q' & China & Dioecious & 22.9 & 90 & 93 & 95 & 95 & 97 & Fast & High \\
\hline 'Han Cold' & China & Dioecious & 20.4 & 21 & 40 & 56 & 59 & 65 & Slow & Low \\
\hline 'Han NE' & China & Dioecious & 21.0 & 24 & 44 & 52 & 54 & 60 & Slow & High \\
\hline 'Han NW' & China & Dioecious & 24.9 & 55 & 63 & 70 & 78 & 81 & Slow & Low \\
\hline ‘Bama’ & China & Dioecious & 31.7 & 38 & 69 & 79 & 81 & 84 & Slow & Medium \\
\hline 'Puma 3' & China & Dioecious & 41.1 & 47 & 68 & 77 & 78 & 83 & Slow & Medium \\
\hline 'Yuma' & China & Dioecious & 31.0 & 44 & 73 & 83 & 87 & 87 & Slow & High \\
\hline 'SI-1' & China & Dioecious & 58.8 & 11 & 17 & 29 & 32 & 33 & Slow & Low \\
\hline
\end{tabular}

Paddocks were sprayed before ploughing with Roundup ${ }^{\circledR}$ (24 l/ha) followed by Hammer ${ }^{\circledR}$ ( $25 \mathrm{ml} / \mathrm{ha}$ ). Fields were ploughed and then multi-disced two or three times prior to broadcasting NPKS 14-16-11-1 fertilizer (350 kg/ha) with sodium borate $14.8 \%(3.0 \mathrm{~kg} / \mathrm{ha})$ at both trial sites. Trial plots were boom sprayed for fallow and pre-emergent weed control with SpraySeed ${ }^{\circledR}(2.5 \mathrm{l} / \mathrm{ha})$ prior to sowing and with Stomp $440^{\circledR}(750 \mathrm{ml} / \mathrm{ha})$ immediately after sowing followed by irrigation. Post emergent control of limited broadleaf weed (ex., fat hen, blackberry nightshade, wild radish, pink weed, hogweed and chickweed) infestations was achieved by pre-irrigation followed by boom spraying with Bromoxicide $200^{\circledR}$ (1 l/ha) prior to canopy closure. All herbicides used are certified for use on hemp seed crops and they were applied at below allowed concentrations.

\subsection{Plot sizes}

In the previous Tasmanian hemp variety trials [4] potential seed yields were extrapolated from small plots harvested by hand. Edge effects (ex., increased vigor, branching, weed pressure, etc.) can be strong especially in small plots with a high ratio of edges. With the exception of 'Han Cold' and 'Si-1' of which we received limited amounts of seed the Bothwell trials were sown in larger plots ( 0.10 to $0.78 \mathrm{ha}$; average $0.28 \mathrm{ha}$ ) to dilute edge effects, and allow harvesting by header combine thereby producing more accurate yield data. Seeds were sown at a depth of approximately 1.5 to 2.0 centimeters using a pneumatic drill carefully calibrated to sow seeds at the predetermined rates.

\subsection{Sowing dates}

Each cultivar has a narrow window of sowing dates that will produce a favorable outcome and determining the optimum sowing date is key to ensuring hemp cultivar performance. Hemp crops require sufficient time to develop root systems supporting rapid vegetative growth and canopy closure prior to flowering and setting seed. If sown too early the crop will grow tall and can become difficult to harvest with conventional equipment, and extended crop cycles also invite more pest and disease problems. When sown too late, hemp seed crops can flower prematurely before the canopy has time to close, fields become choked with weeds and seed yields are reduced.

Four sowing dates were planned for the Bothwell trials, but due to late arrival of sowing seed plots were sown in three groups (11/28-29/2018, 12/22-23/2018 and 1/9-10/2019). The third sowing was still in its juvenile growth stage at the time the project was terminated, and only limited data is reported. Two sowing dates allow only two possible outcomes. Either one of the two sowing dates appears to be optimum (one will always be the better of the two) or we can conclude that we should have sown earlier or later, but cannot accurately determine by how many weeks earlier or later. An additional earlier sowing would have allowed a more accurate determination of the optimum sowing date for each cultivar.

The Canadian varieties were only sown during the second round (12/22-23/2018) because the seed arrived too late 
for the first sowing and sowing them in the third round was predicted to result in premature flowering and seed set. The French and Chinese cultivars were sown three times (11/28-29/2018, 12/22-23/2018 and 1/9-10/2019).

\subsection{Seed viability, sowing rate and trial plot areas}

Another key factor influencing crop structure and yield is the sowing rate $(\mathrm{kg} / \mathrm{ha})$ which directly determines how many seedlings emerge and the initial crop density (Table 3). Sowing rate is the third variable in the variety trial in addition to cultivar origin and sowing date. The generally recommended hemp grain crop sowing rate is approximately 35 kilograms per hectare of viable seed. Sowing fewer seeds does not allow sufficient crop density for complete canopy closure and weed suppression.

Table 3. Cultivars, seed weights, germination percentages, viable seeds per kilogram and sowing rates-Target crop density of 100-150 plants per square meter (2018-2019)

\begin{tabular}{|c|c|c|c|c|c|c|c|c|}
\hline Cultivars & $\begin{array}{c}1000 \text { Seed } \\
\text { Weight } \\
(\mathrm{gr})\end{array}$ & $\begin{array}{c}\text { Single Seed } \\
\text { Weight } \\
(\mathrm{gr})\end{array}$ & $\begin{array}{l}\text { Seeds per } \\
\text { kilogram } \\
\text { (approx.) }\end{array}$ & $\begin{array}{c}\text { Germination } \\
\text { Percentage }\end{array}$ & $\begin{array}{c}\text { Viable } \\
\text { Seeds per } \\
\text { kilogram } \\
\text { (approx.) }\end{array}$ & $\underset{\left(\mathrm{gr} / \mathrm{m}^{2}\right)}{\text { Sowing Rate }}$ & $\begin{array}{c}\text { Sowing Rate } \\
@ 150 \\
\text { plants } / \mathrm{m}^{2} \\
\text { (kg/ha) }\end{array}$ & $\begin{array}{c}\text { Sowing Rate } \\
\text { @ } 200 \\
\text { plants } / \mathrm{m}^{2} \\
\text { (kg/ha) }\end{array}$ \\
\hline $\begin{array}{l}\text { 'CFX-1' } \\
\text { 'CRS-1' } \\
\text { 'Katani' } \\
\text { 'Picolo' }\end{array}$ & $\begin{array}{l}17.0 \\
17.8 \\
16.2 \\
16.5\end{array}$ & $\begin{array}{l}.0170 \\
.0178 \\
.0162 \\
.0165\end{array}$ & $\begin{array}{l}59,000 \\
56,000 \\
62,000 \\
61,000\end{array}$ & $\begin{array}{l}90 \\
94 \\
97 \\
84\end{array}$ & $\begin{array}{l}53,000 \\
52,500 \\
60,000 \\
51,000\end{array}$ & $\begin{array}{l}2.94 \\
2.87 \\
2.64 \\
3.12\end{array}$ & $\begin{array}{l}29 \\
29 \\
26 \\
31\end{array}$ & $\begin{array}{l}: \\
:\end{array}$ \\
\hline $\begin{array}{l}\text { 'Felina 32' } \\
\text { 'Ferimon' } \\
\text { ‘Futura 75' } \\
\text { 'Santhica 70' }\end{array}$ & $\begin{array}{l}17.8 \\
17.1 \\
18.8 \\
18.1\end{array}$ & $\begin{array}{l}.0178 \\
.0171 \\
.0188 \\
.0181\end{array}$ & $\begin{array}{l}56,000 \\
58,500 \\
53,000 \\
55,000\end{array}$ & $\begin{array}{l}95 \\
94 \\
89 \\
94\end{array}$ & $\begin{array}{l}53,000 \\
55,000 \\
47,000 \\
51,500\end{array}$ & $\begin{array}{l}2.81 \\
2.73 \\
3.17 \\
2.89\end{array}$ & $\begin{array}{l}28 \\
27 \\
32 \\
29\end{array}$ & $\begin{array}{l}- \\
- \\
-\end{array}$ \\
\hline $\begin{array}{l}\text { 'Han FN H' } \\
\text { 'Han FN Q' } \\
\text { 'Han Cold' }\end{array}$ & $\begin{array}{l}22.2 \\
22.9 \\
20.4\end{array}$ & $\begin{array}{l}.0222 \\
.0229 \\
.0204\end{array}$ & $\begin{array}{l}45,000 \\
43,500 \\
49,000\end{array}$ & $\begin{array}{l}62 \\
97 \\
65\end{array}$ & $\begin{array}{l}28,000 \\
42,500 \\
32,000\end{array}$ & $\begin{array}{l}7.16 \\
3.33 \\
6.28\end{array}$ & 33 & $\begin{array}{c}72 \\
- \\
63\end{array}$ \\
\hline $\begin{array}{l}\text { ‘Han NE' } \\
\text { 'Han NW' }\end{array}$ & $\begin{array}{l}21.0 \\
24.9\end{array}$ & $\begin{array}{l}.0210 \\
.0249\end{array}$ & $\begin{array}{l}47,500 \\
40,000\end{array}$ & $\begin{array}{l}81 \\
60\end{array}$ & $\begin{array}{l}38,500 \\
24,000\end{array}$ & $\begin{array}{l}3.89 \\
8.30\end{array}$ & 39 & 83 \\
\hline $\begin{array}{l}\text { 'Bama' } \\
\text { 'Puma-3' } \\
\text { 'Yuma' } \\
\text { 'Si-1' }\end{array}$ & $\begin{array}{l}31.7 \\
41.1 \\
31.0 \\
58.8\end{array}$ & $\begin{array}{l}.0317 \\
.0411 \\
.0310 \\
.0588\end{array}$ & $\begin{array}{l}31,500 \\
24,500 \\
32,000 \\
17,000\end{array}$ & $\begin{array}{l}84 \\
83 \\
87 \\
33\end{array}$ & $\begin{array}{c}26,500 \\
20,500 \\
28,000 \\
5600\end{array}$ & $\begin{array}{c}5.66 \\
7.43 \\
5.34 \\
26.46\end{array}$ & $\begin{array}{l}57 \\
74 \\
53 \\
-\end{array}$ & $\begin{array}{c}- \\
- \\
- \\
260\end{array}$ \\
\hline
\end{tabular}

The Canadian cultivars were sown only once while seeds of the French and Chinese cultivars were divided into thirds to be equally represented in each sowing. The size of each trial plot was determined by the amount of seed available and the viability of that seed. The Chinese trial plots were generally the largest, followed by the Canadian and French, and all plots were large enough to be harvested with a combine (Tables 4, 5, and 6).

Table 4. Trial sowings $1-$ Cultivars, seed sowing rates and sown areas (November 28-29, 2018)

\begin{tabular}{|c|c|c|c|c|c|c|c|c|c|c|}
\hline Cultivars & $\begin{array}{c}\text { Sowing } \\
\text { rates } \\
(\mathrm{kg} / \mathrm{ha}) \\
@ 150 \\
\text { plants/m² }\end{array}$ & $\begin{array}{c}\text { Sowing } \\
\text { rates } \\
\text { (kg/ha) } \\
\text { @ } 200 \\
\text { plants/m² }\end{array}$ & $\begin{array}{l}\text { Proposed } \\
\text { Sowing } \\
\text { areas } \\
\text { Edgell } \\
\text { (ha) }\end{array}$ & $\begin{array}{l}\text { Actual } \\
\text { Areas } \\
\text { sown } \\
\text { Edgell } \\
\text { (Drill data) } \\
\text { (ha) }\end{array}$ & $\begin{array}{l}\text { Plot } \\
\text { Nos. } \\
\text { Edgell }\end{array}$ & $\begin{array}{c}\text { Seed } \\
\text { Sown } \\
\text { Edgell } \\
(\mathrm{kg})\end{array}$ & $\begin{array}{l}\text { Proposed } \\
\text { Sowing } \\
\text { areas } \\
\text { Ramsay } \\
\text { (ha) }\end{array}$ & $\begin{array}{c}\text { Actual } \\
\text { Areas } \\
\text { sown } \\
\text { Ramsay } \\
\text { (Drill data) } \\
\text { (ha) }\end{array}$ & $\begin{array}{c}\text { Plot } \\
\text { Nos. } \\
\text { Ramsay }\end{array}$ & $\begin{array}{c}\text { Seed } \\
\text { Sown } \\
\text { Ramsay } \\
(\mathrm{kg})\end{array}$ \\
\hline 'Felina 32' & 28 & - & 0.15 & 0.13 & $3 d$ & 4.0 & 0.15 & 0.16 & $7 c$ & 4.0 \\
\hline ‘Ferimon' & 27 & - & 0.15 & 0.15 & $3 a$ & 4.0 & 0.15 & 0.16 & $7 a$ & 4.0 \\
\hline ‘Futura 75’ & 32 & - & 0.15 & 0.13 & $3 c$ & 4.0 & 0.15 & 0.13 & 3 & 4.0 \\
\hline 'Santhica 70' & 29 & - & 0.15 & 0.14 & $3 b$ & 4.0 & 0.15 & 0.16 & $7 b$ & 4.0 \\
\hline ‘Han FN H' & - & 72 & 0.37 & 0.37 & $5 e$ & 26.5 & 0.37 & 0.39 & 6 & 26.5 \\
\hline 'Han FN Q' & 33 & - & 0.80 & 0.67 & 4 & 26.5 & 0.80 & 0.75 & 10 & 26.5 \\
\hline ‘Han NE’ & - & 83 & 0.20 & 0.23 & $5 d$ & 16.5 & 0.20 & 0.20 & $9 b$ & 16.5 \\
\hline 'Han NW' & 39 & - & 0.43 & 0.42 & 2 & 16.5 & 0.43 & 0.38 & 8 & 16.5 \\
\hline 'Bama' & 57 & - & 0.30 & 0.30 & $5 b$ & 16.5 & 0.30 & 0.29 & 5 & 16.5 \\
\hline 'Puma 3' & 74 & - & 0.23 & 0.22 & $5 a$ & 16.5 & 0.23 & 0.24 & 4 & 16.5 \\
\hline 'Yuma' & 53 & - & 0.32 & 0.29 & $5 c$ & 16.5 & 0.32 & 0.31 & $9 a$ & 16.5 \\
\hline 'Si-1' & - & 356 & 0.05 & 0.05 & 1 & 16.5 & 0.05 & 0.05 & 1 & 16.5 \\
\hline \multicolumn{3}{|c|}{ Planned sown area $=6.6 \mathrm{ha}$} & $3.3 \mathrm{ha}$ & & & & 3.3 ha & & & \\
\hline \multicolumn{3}{|c|}{ Actual sown area $=6.32 \mathrm{ha}$} & & 3.10 ha & & & & 3.22 ha & & \\
\hline
\end{tabular}


Table 5. Trial sowings 2-Cultivars, seed sowing rates and sown areas (December 22-23, 2018)

\begin{tabular}{|c|c|c|c|c|c|c|c|c|c|c|}
\hline Cultivars & $\begin{array}{c}\text { Sowing rates } \\
\text { (kg/ha) } \\
@ 150 \\
\text { plants } / \mathrm{m}^{2}\end{array}$ & $\begin{array}{c}\text { Sowing rates } \\
(\mathrm{kg} / \mathrm{ha}) \\
@ 200 \\
\text { plants } / \mathrm{m}^{2}\end{array}$ & $\begin{array}{l}\text { Proposed } \\
\text { Sowing } \\
\text { areas } \\
\text { Edgell } \\
\text { (ha) }\end{array}$ & $\begin{array}{c}\text { Actual } \\
\text { Areas sown } \\
\text { Edgell } \\
\text { (Drill data) } \\
\text { (ha) }\end{array}$ & $\begin{array}{l}\text { Plot } \\
\text { Nos. } \\
\text { Edgell }\end{array}$ & $\begin{array}{l}\text { Seed } \\
\text { Sown } \\
\text { Edgell } \\
(\mathrm{kg})\end{array}$ & $\begin{array}{l}\text { Proposed } \\
\text { Sowing } \\
\text { areas } \\
\text { Ramsay } \\
\text { (ha) }\end{array}$ & $\begin{array}{c}\text { Actual } \\
\text { Areas sown } \\
\text { Ramsay } \\
\text { (Drill data) } \\
\text { (ha) }\end{array}$ & $\begin{array}{c}\text { Plot } \\
\text { Nos. } \\
\text { Ramsay }\end{array}$ & $\begin{array}{c}\text { Seed } \\
\text { Sown } \\
\text { Ramsay } \\
(\mathrm{kg})\end{array}$ \\
\hline ‘CFX-1’' & 29 & - & 0.50 & 0.27 & 9 & 13.7 & 0.80 & 0.78 & 14 & 17.7 \\
\hline 'CRS-1' & 29 & - & 0.50 & 0.63 & 8 & 21.5 & 0.50 & 0.64 & 13 & 22.7 \\
\hline ‘Katani’ & 26 & - & 0.50 & 0.35 & 6 & 11.3 & 0.50 & 0.44 & 11 & 15.4 \\
\hline ‘Picolo' & 31 & - & 0.50 & 0.71 & 7 & 22.7 & 0.50 & 0.53 & 12 & 18.8 \\
\hline 'Felina 32' & 28 & - & 0.15 & 0.16 & 3d & 4.0 & 0.15 & 0.10 & $7 c$ & 4.0 \\
\hline 'Ferimon' & 27 & - & 0.15 & 0.13 & $3 a$ & 4.0 & 0.15 & 0.12 & $7 a$ & 4.0 \\
\hline 'Futura 75' & 32 & - & 0.15 & 0.14 & $3 c$ & 4.0 & 0.15 & 0.12 & 3 & 4.0 \\
\hline 'Santhica 70' & 29 & - & 0.15 & 0.14 & $3 b$ & 4.0 & 0.15 & 0.13 & $7 b$ & 4.0 \\
\hline 'Han FN H' & - & 72 & 0.37 & 0.17 & $5 e$ & 12.5 & 0.37 & 0.43 & 6 & 26.5 \\
\hline 'Han FN Q' & 33 & - & 0.50 & 0.33 & 4 & 14.0 & 0.80 & 0.63 & 10 & 26.5 \\
\hline 'Han Cold' & $\cdot$ & 63 & 0.05 & 0.07 & $1 b$ & 3.1 & 0.05 & 0.10 & 2 & 3.1 \\
\hline 'Han NE' & - & 83 & 0.20 & 0.16 & $5 d$ & 16.5 & 0.20 & 0.27 & $9 b$ & 16.5 \\
\hline 'Han NW' & 39 & $\cdot$ & 0.43 & 0.34 & 2 & 16.5 & 0.43 & 0.36 & 8 & 16.5 \\
\hline 'Bama' & 57 & - & 0.30 & 0.20 & $5 b$ & 16.5 & 0.30 & 0.25 & 5 & 16.5 \\
\hline 'Puma 3' & 74 & - & 0.23 & 0.13 & $5 a$ & 9.7 & 0.23 & 0.23 & 4 & 16.5 \\
\hline 'Yuma' & 53 & - & 0.32 & 0.16 & $5 c$ & 9.2 & 0.32 & 0.32 & $9 a$ & 16.5 \\
\hline 'Si-1' & - & 356 & 0.05 & 0.05 & 1 & 16.5 & 0.05 & 0.05 & 1 & 16.5 \\
\hline \multicolumn{3}{|c|}{ Proposed area $=10.7$ ha Total } & 5.05 ha & & & & 5.65 ha & & & \\
\hline \multicolumn{3}{|c|}{ Actual area $=9.37$ ha Total } & & 4.14 ha & & & & 5.23 ha & & \\
\hline
\end{tabular}

Table 6. Trial sowings 3-Cultivars, seed sowing rates and sown areas (January 9-10, 2019)

\begin{tabular}{|c|c|c|c|c|c|c|c|c|c|c|}
\hline Cultivars & $\begin{array}{c}\text { Sowing } \\
\text { rates } \\
(\mathrm{kg} / \mathrm{ha}) \\
@ 150 \\
\text { plants } / \mathrm{m}^{2}\end{array}$ & $\begin{array}{c}\text { Sowing } \\
\text { rates } \\
(\mathrm{kg} / \mathrm{ha}) \\
@ 200 \\
\text { plants/m² }\end{array}$ & $\begin{array}{l}\text { Proposed } \\
\text { Sowing } \\
\text { Areas } \\
\text { Edgell } \\
\text { (ha) }\end{array}$ & $\begin{array}{l}\text { Actual } \\
\text { Areas } \\
\text { sown } \\
\text { Edgell } \\
\text { (Drill data) } \\
\text { (ha) }\end{array}$ & $\begin{array}{l}\text { Plot } \\
\text { Nos. } \\
\text { Edgell }\end{array}$ & $\begin{array}{c}\text { Seed } \\
\text { sown } \\
\text { Edgell } \\
(\mathrm{kg})\end{array}$ & $\begin{array}{l}\text { Proposed } \\
\text { Sowing } \\
\text { Areas } \\
\text { Ramsay } \\
\text { (ha) }\end{array}$ & $\begin{array}{c}\text { Actual } \\
\text { Areas } \\
\text { sown } \\
\text { Ramsay } \\
\text { (Drill data) } \\
\text { (ha) }\end{array}$ & $\begin{array}{c}\text { Plot } \\
\text { Nos. } \\
\text { Ramsay }\end{array}$ & $\begin{array}{c}\text { Seed } \\
\text { sown } \\
\text { Ramsay } \\
(\mathrm{kg})\end{array}$ \\
\hline 'Felina 32' & 28 & - & 0.15 & 0.15 & 4 & 3.5 & 0.15 & 0.12 & 8 & 3.5 \\
\hline ‘Ferimon' & 27 & - & 0.15 & 0.14 & 1 & 3.5 & 0.15 & 0.13 & 6 & 3.5 \\
\hline 'Futura 75’ & 32 & - & 0.15 & 0.15 & 3 & 3.5 & 0.15 & 0.10 & 2 & 3.5 \\
\hline 'Santhica 70' & 29 & - & 0.15 & 0.15 & 2 & 3.5 & 0.15 & 0.14 & 7 & 3.5 \\
\hline ‘Han FN H’ & - & 72 & 0.37 & 0.54 & 9 & 26.5 & 0.37 & 0.14 & 5 & 26.5 \\
\hline 'Han FN Q' & 33 & $\cdot$ & 0.50 & 0.23 & 11 & 26.5 & 0.50 & 0.17 & 12 & 26.5 \\
\hline ‘Han NE' & - & 83 & 0.20 & 0.27 & 5 & 16.5 & 0.20 & 0.28 & 11 & 16.5 \\
\hline 'Han NW' & 39 & - & 0.43 & 0.38 & 10 & 16.5 & 0.43 & 0.36 & 9 & 16.5 \\
\hline 'Bama' & 57 & - & 0.30 & 0.30 & 7 & 16.5 & 0.30 & 0.11 & 4 & 16.5 \\
\hline 'Puma 3' & 74 & - & 0.23 & 0.62 & 6 & 16.5 & 0.23 & 0.23 & 3 & 16.5 \\
\hline 'Yuma' & 53 & - & 0.32 & 0.31 & 8 & 16.5 & 0.32 & 0.33 & 10 & 16.5 \\
\hline 'Si-1' & - & 356 & 0.05 & 0.05 & 12 & 16.5 & 0.05 & 0.05 & 1 & 16.5 \\
\hline \multicolumn{3}{|c|}{ Proposed area $=6.0$ ha Total } & 3.0 ha & & & & 3.0 ha & & & \\
\hline \multicolumn{3}{|c|}{ Actual area $=5.45$ ha Total } & & 3.29 ha & & & & 2.16 ha & & \\
\hline
\end{tabular}

\subsection{Irrigation}

Although rainfall may occur throughout the summer and early autumn, precipitation supplies only about one-third of the water required and is insufficient to adequately support a healthy hemp crop and produce economically viable yields. Hemp grows well in sandier soils that require additional irrigation. Tasmanian Irrigation suggests that hemp crops require 2-3 ML/ha of water per crop cycle [5]. The South Australia trials of mixed varieties estimated their water use at 4-6 ML/ha [6]. The relatively sandy and porous soils of the Bothwell trial sites required water availability of 4-5 ML/ha.

\subsection{Data gathering}

Fields were monitored as the trial plots matured and observational data were collected approximately every two weeks. Early in the crop cycle plots were monitored for vegetative vigor, node development, height and canopy closure. Once flowering commenced, dates of first pollen release, seed set and seed maturity were recorded. Plots were also monitored for levels of pest infestation as well as THC levels. Seed yields were determined after the seed was harvested, dried and cleaned. 


\section{Results}

\subsection{Weed and pest control}

Weeds in hemp fields are most effectively controlled through rapid canopy establishment encouraged by locally appropriate field practices. Pre-emergent herbicides were applied to the fields at the time of sowing and again prior to full canopy closure. In limited areas of trial blocks with lower plant densities and incomplete canopy cover some weeds gained a foothold but their presence resulted in little if any loss of yield. Observed early-on during crop establishment were Chenopodium and fat hen with additional anticipated weeds appearing during seed set especially in the shorter Canadian cultivars. Taller French and Chinese cultivars were unaffected by weeds except along the perimeters of the trial plots where edge effects were apparent.

Hemp crops attract a diverse array of organisms both beneficial and potentially damaging and their naturally low populations remain in balance in healthy fields. However, stressed hemp crops subjected to adverse conditions can attract pests in surprising numbers and the trial plots were carefully monitored for signs of potential pest infestations. Early on (January 16, 2019) insect feeding signs were observed in the first-sown plots as thrip and grasshopper infestations caused minimal leaf damage. Heliothis winged adults annually disperse from the Australian mainland carried by the northwest winds, but they were rarely recorded at the Bothwell trial sites. Heliothis larvae destroyed some seeds at grain filling stage and caused limited collateral damage to tissues within the same inflorescence, but infestations did not reach economic thresholds.

\subsection{Crop growth and development-Vegetative stages}

The Bothwell hemp cultivar trials performed largely as expected with no damaging weather conditions and away from local brush fires. Approximately two weeks following the first sowing (December 12, 2019) seedlings reached the second leaf stage and the four French cultivar plots appeared uniform with only a few gaps of exposed ground in the plots. Three weeks later (January 2, 2019) canopies were closing in the first-sown French and Chinese plots and the second sowings including the four Canadian seed cultivars had reached the second leaf stage. The Canadian cultivars were beginning to prematurely flower, but they continued to develop more nodes and elongate rapidly well into seed set reaching final height ranges of $120-160 \mathrm{~cm}$ at the $\mathrm{E}$ site and $80-180 \mathrm{~cm}$ at the $\mathrm{R}$ site (Table 7).

By January 16, 2019, the first sowing of French cultivars reached 120-170 cm at the E site and 90-150 cm at the R site as flowering commenced. By January 29 the second sowing of French cultivars reached 35-60 cm at the E site and 20-40 $\mathrm{cm}$ at the $\mathrm{R}$ site (Table 7).

Table 7. Crop development stages—Vegetative—Canadian and French cultivars (2018-2019)

\begin{tabular}{|c|c|c|c|c|c|c|c|c|c|c|c|c|c|c|c|}
\hline Observation Date & Field & Sowing Date & $\begin{array}{l}\text { Nodes } \\
12 / 12 / 2018\end{array}$ & 0102/2019 & $\begin{array}{c}\text { Crop } \\
\text { Height } \\
\text { (cm) } \\
01 / 02 / 2019\end{array}$ & 01/16/2019 & $\begin{array}{c}\text { Crop } \\
\text { Height } \\
\text { (cm) } \\
01 / 16 / 2019\end{array}$ & 01/22/2018 & $\begin{array}{c}\text { Crop } \\
\text { Height } \\
\text { (cm) } \\
01 / 22 / 2019\end{array}$ & 01/29/2018 & $\begin{array}{c}\text { Crop } \\
\text { Height } \\
\text { (cm) } \\
01 / 29 / 2019\end{array}$ & $\begin{array}{l}\text { Nodes } \\
02066 / 2019\end{array}$ & $\begin{array}{c}\text { Crop } \\
\text { Height } \\
(\mathrm{cm}) \\
02106 / 2019\end{array}$ & $\begin{array}{l}\text { Nodes } \\
02 / 13 / 2019\end{array}$ & $\begin{array}{c}\text { Crop } \\
\text { Height } \\
(\mathrm{cm}) \\
02 / 13 / 2019\end{array}$ \\
\hline $\begin{array}{l}\text { 'CFX-1' } \\
\text { 'CFX-1' }\end{array}$ & $\begin{array}{l}\text { R2 } \\
\text { E2 }\end{array}$ & $\begin{array}{l}12 / 22 / 2018 \\
12 / 23 / 2018\end{array}$ & & $\begin{array}{l}2^{\text {nd }} \text { leaf } \\
2^{\text {nd }} \text { leaf }\end{array}$ & $\begin{array}{l}10 \\
10\end{array}$ & $\begin{array}{l}5-6 \\
5-6\end{array}$ & $\begin{array}{l}20-30 \\
50-60\end{array}$ & $\begin{array}{l}5-6 \\
5-6\end{array}$ & $\begin{array}{l}40-50 \\
70-80\end{array}$ & $\begin{array}{l}5-7 \\
5-7\end{array}$ & $\begin{array}{c}60-80 \\
80-100\end{array}$ & $\begin{array}{l}6-7 \\
6-7\end{array}$ & $\begin{array}{c}80-120 \\
100-140\end{array}$ & $\begin{array}{l}7-8 \\
7-8\end{array}$ & $\begin{array}{l}110-150 \\
120-160\end{array}$ \\
\hline $\begin{array}{l}\text { 'CRS-1' } \\
\text { 'CRS-1' }\end{array}$ & $\begin{array}{l}\text { R2 } \\
\text { E2 }\end{array}$ & $\begin{array}{l}12 / 22 / 2018 \\
12 / 23 / 2018\end{array}$ & & $\begin{array}{l}2^{\text {nd }} \text { leaf } \\
2^{\text {nd }} \text { leaf }\end{array}$ & $\begin{array}{l}10 \\
10\end{array}$ & $\begin{array}{l}5-6 \\
5-6\end{array}$ & $\begin{array}{l}20-40 \\
25-40\end{array}$ & $\begin{array}{l}5-7 \\
5-7\end{array}$ & $\begin{array}{l}50-70 \\
50-70\end{array}$ & $\begin{array}{l}5-7 \\
5-7\end{array}$ & $\begin{array}{l}70-90 \\
70-90\end{array}$ & $\begin{array}{l}6-7 \\
6-7\end{array}$ & $\begin{array}{c}90-130 \\
100-150\end{array}$ & $\begin{array}{l}8-10 \\
8-10\end{array}$ & $\begin{array}{l}150-180 \\
140-160\end{array}$ \\
\hline $\begin{array}{l}\text { 'Katani' } \\
\text { 'Katani' }\end{array}$ & $\begin{array}{l}\text { R2 } \\
\text { E2 }\end{array}$ & $\begin{array}{l}12 / 22 / 2018 \\
12 / 23 / 2018\end{array}$ & & $\begin{array}{l}2^{\text {nd }} \text { leaf } \\
2^{\text {nd }} \text { leaf }\end{array}$ & $\begin{array}{l}10 \\
10\end{array}$ & $\begin{array}{l}5-6 \\
5-6\end{array}$ & $\begin{array}{l}20-30 \\
50-60\end{array}$ & $\begin{array}{l}5-7 \\
5-7\end{array}$ & $\begin{array}{l}40-70 \\
40-70\end{array}$ & $\begin{array}{l}5-7 \\
5-7\end{array}$ & $\begin{array}{l}70-90 \\
70-90\end{array}$ & $\begin{array}{l}6-7 \\
6-7\end{array}$ & $\begin{array}{l}100-120 \\
100-140\end{array}$ & $\begin{array}{l}7-8 \\
7-8\end{array}$ & $\begin{array}{l}100-140 \\
120-160\end{array}$ \\
\hline $\begin{array}{l}\text { 'Picolo' } \\
\text { 'Picolo' }\end{array}$ & $\begin{array}{l}\text { R2 } \\
\text { E2 }\end{array}$ & $\begin{array}{l}12 / 22 / 2018 \\
12 / 23 / 2018\end{array}$ & & $\begin{array}{l}2^{\text {nd }} \text { leaf } \\
2^{\text {nd }} \text { leaf }\end{array}$ & $\begin{array}{l}10 \\
10\end{array}$ & $\begin{array}{l}5-6 \\
5-6\end{array}$ & $\begin{array}{l}30-40 \\
40-60\end{array}$ & $\begin{array}{l}5-7 \\
57\end{array}$ & $\begin{array}{l}40-70 \\
40-70\end{array}$ & $\begin{array}{l}5-7 \\
5-7\end{array}$ & $\begin{array}{l}70-90 \\
70-90\end{array}$ & $\begin{array}{l}6-7 \\
6-7\end{array}$ & $\begin{array}{c}90-110 \\
100-120\end{array}$ & $\begin{array}{c}7-8 \\
8-10\end{array}$ & $\begin{array}{c}80-120 \\
140-160\end{array}$ \\
\hline $\begin{array}{l}\text { 'Felina 32' } \\
\text { 'Felina 32' }\end{array}$ & $\begin{array}{l}\mathrm{R} 1 \\
\mathrm{E} 1\end{array}$ & $\begin{array}{l}11 / 28 / 2018 \\
11 / 29 / 2018\end{array}$ & $\begin{array}{l}2^{\text {nd }} \text { le af } \\
2^{\text {nd }} \text { le af }\end{array}$ & $\begin{array}{l}5 \\
6\end{array}$ & $\begin{array}{l}50-60 \\
60-70\end{array}$ & $\begin{array}{c}10-11 \\
8-10\end{array}$ & $\begin{array}{c}90-110 \\
150-160\end{array}$ & $\begin{array}{c}10-11 \\
8-10\end{array}$ & $\begin{array}{l}100-120 \\
160-180\end{array}$ & $\begin{array}{c}10-11 \\
8-10\end{array}$ & $\begin{array}{l}100-130 \\
170-200\end{array}$ & $\begin{array}{c}10-12 \\
9-11\end{array}$ & $\begin{array}{l}110-140 \\
170-210\end{array}$ & $\begin{array}{l}11-12 \\
12-14\end{array}$ & $\begin{array}{l}150-200 \\
180-220\end{array}$ \\
\hline $\begin{array}{l}\text { 'Felina 32' } \\
\text { 'Felina 32' }\end{array}$ & $\begin{array}{l}\text { R2 } \\
\text { E2 }\end{array}$ & $\begin{array}{l}12 / 22 / 2018 \\
12 / 23 / 2018\end{array}$ & & $\begin{array}{l}2^{\text {nd }} \text { leaf } \\
1^{\text {st }} \text { leaf }\end{array}$ & $\begin{array}{l}10 \\
10\end{array}$ & $\begin{array}{l}4-6 \\
5-6\end{array}$ & $\begin{array}{l}20-30 \\
35-45\end{array}$ & $\begin{array}{l}5-7 \\
6-8\end{array}$ & $\begin{array}{l}40-70 \\
70-100\end{array}$ & $\begin{array}{c}5-7 \\
8-10\end{array}$ & $\begin{array}{c}70-90 \\
110-150\end{array}$ & $\begin{array}{l}8-9 \\
9-11\end{array}$ & $\begin{array}{l}110-130 \\
160-200\end{array}$ & $\begin{array}{c}8-10 \\
10-12\end{array}$ & $\begin{array}{l}140-160 \\
150-180\end{array}$ \\
\hline 'Felina 32' & R3 & $01 / 09 / 2019$ & & & & & & & & & & & & $6-7$ & $40-50$ \\
\hline $\begin{array}{l}\text { 'Ferimon' } \\
\text { 'Ferimon' }\end{array}$ & $\begin{array}{l}\text { R1 } \\
\text { E1 }\end{array}$ & $\begin{array}{l}11 / 28 / 2018 \\
11 / 29 / 2018\end{array}$ & $\begin{array}{l}2^{\text {nd }} \text { le af } \\
2^{\text {nd }} \text { le af }\end{array}$ & $\begin{array}{l}6 \\
5\end{array}$ & $\begin{array}{l}50-60 \\
40-50\end{array}$ & $\begin{array}{c}9-11 \\
10-12\end{array}$ & $\begin{array}{l}130-150 \\
140-170\end{array}$ & $\begin{array}{c}9-11 \\
10-12\end{array}$ & $\begin{array}{l}140-170 \\
140-180\end{array}$ & $\begin{array}{c}9-11 \\
10-13\end{array}$ & $\begin{array}{l}150-180 \\
150-180\end{array}$ & $\begin{array}{c}9-11 \\
11-13\end{array}$ & $\begin{array}{l}150-200 \\
150-200\end{array}$ & $\begin{array}{l}10-12 \\
12-14\end{array}$ & $\begin{array}{l}160-230 \\
180-220\end{array}$ \\
\hline $\begin{array}{l}\text { 'Ferimon' } \\
\text { 'Ferimon' }\end{array}$ & $\begin{array}{l}\text { R2 } \\
\text { E2 }\end{array}$ & $\begin{array}{l}12 / 22 / 2018 \\
12 / 23 / 2018\end{array}$ & & $\begin{array}{l}2^{\text {nd }} \text { leaf } \\
1^{\text {st }} \text { leaf }\end{array}$ & $\begin{array}{l}10 \\
10\end{array}$ & $\begin{array}{l}5-6 \\
5-6\end{array}$ & $\begin{array}{l}30-40 \\
45-55\end{array}$ & $\begin{array}{c}5-7 \\
8-10\end{array}$ & $\begin{array}{l}40-70 \\
60-80\end{array}$ & $\begin{array}{c}5-7 \\
8-10\end{array}$ & $\begin{array}{c}70-90 \\
80-100\end{array}$ & $\begin{array}{c}8-9 \\
9-11\end{array}$ & $\begin{array}{l}100-120 \\
100-170\end{array}$ & $\begin{array}{c}8-10 \\
12-14\end{array}$ & $\begin{array}{l}120-160 \\
160-200\end{array}$ \\
\hline 'Ferimon' & R3 & 01/09/2019 & & & & & & & & & & & & $6-7$ & $40-50$ \\
\hline $\begin{array}{l}\text { 'Futura 75' } \\
\text { 'Futura 75' }\end{array}$ & $\begin{array}{l}\text { R1 } \\
\text { E1 }\end{array}$ & $\begin{array}{l}11 / 28 / 2018 \\
11 / 29 / 2018\end{array}$ & $\begin{array}{l}2^{\text {nd }} \text { le af } \\
2^{\text {nd }} \text { le af }\end{array}$ & $\begin{array}{l}5 \\
5\end{array}$ & $\begin{array}{l}40-50 \\
60-70\end{array}$ & $\begin{array}{l}8-10 \\
8-10\end{array}$ & $\begin{array}{l}130-150 \\
120-150\end{array}$ & $\begin{array}{l}8-10 \\
8-10\end{array}$ & $\begin{array}{l}140-160 \\
130-160\end{array}$ & $\begin{array}{l}8-10 \\
8-10\end{array}$ & $\begin{array}{l}150-190 \\
140-180\end{array}$ & $\begin{array}{l}9-11 \\
9-11\end{array}$ & $\begin{array}{l}160-210 \\
150-210\end{array}$ & $\begin{array}{l}10-12 \\
12-14\end{array}$ & $\begin{array}{l}200-240 \\
220-250\end{array}$ \\
\hline $\begin{array}{l}\text { 'Futura 75' } \\
\text { 'Futura 75' }\end{array}$ & $\begin{array}{l}\mathrm{R} 2 \\
\mathrm{E} 2\end{array}$ & $\begin{array}{l}12 / 22 / 2018 \\
12 / 23 / 2018\end{array}$ & & $\begin{array}{l}2^{\text {nd }} \text { leaf } \\
1^{\text {st }} \text { leaf }\end{array}$ & $\begin{array}{l}10 \\
10\end{array}$ & $\begin{array}{l}4 \\
6\end{array}$ & $\begin{array}{l}20-30 \\
45-55\end{array}$ & $\begin{array}{c}7-8 \\
8-10\end{array}$ & $\begin{array}{l}50-70 \\
60-80\end{array}$ & $\begin{array}{c}8-9 \\
8-10\end{array}$ & $\begin{array}{l}80-100 \\
80-100\end{array}$ & $\begin{array}{l}8-9 \\
8-9\end{array}$ & $\begin{array}{l}110-130 \\
100-150\end{array}$ & $\begin{array}{l}12-14 \\
12-14\end{array}$ & $\begin{array}{l}120-140 \\
180-220\end{array}$ \\
\hline 'Futura 75' & R3 & $01 / 09 / 2019$ & & & & & & & & & & & & $6-7$ & $30-50$ \\
\hline $\begin{array}{l}\text { 'Santhica 70' } \\
\text { 'Santhica 70' }\end{array}$ & $\begin{array}{l}\text { R1 } \\
\text { E1 }\end{array}$ & $\begin{array}{l}11 / 28 / 2018 \\
11 / 29 / 2018\end{array}$ & $\begin{array}{l}2^{\text {nd }} \text { le af } \\
2^{\text {nd }} \text { le af }\end{array}$ & $\begin{array}{l}6 \\
6\end{array}$ & $\begin{array}{l}50-60 \\
50-60\end{array}$ & $\begin{array}{c}10-12 \\
8-10\end{array}$ & $\begin{array}{l}100-120 \\
130-140\end{array}$ & $\begin{array}{c}10-12 \\
8-10\end{array}$ & $\begin{array}{l}110-150 \\
140-170\end{array}$ & $\begin{array}{c}10-12 \\
8-10\end{array}$ & $\begin{array}{l}140-190 \\
150-180\end{array}$ & $\begin{array}{l}9-11 \\
9-11\end{array}$ & $\begin{array}{l}160-210 \\
160-210\end{array}$ & $\begin{array}{l}10-12 \\
12-14\end{array}$ & $\begin{array}{l}180-220 \\
180-240\end{array}$ \\
\hline $\begin{array}{l}\text { 'Santhica 70' } \\
\text { 'Santhica 70' }\end{array}$ & $\begin{array}{l}\text { R2 } \\
\text { E2 }\end{array}$ & $\begin{array}{l}12 / 22 / 2018 \\
12 / 23 / 2018\end{array}$ & & $\begin{array}{l}2^{\text {nd }} \text { leaf } \\
1^{\text {st }} \text { leaf }\end{array}$ & $\begin{array}{l}10 \\
10\end{array}$ & $\begin{array}{c}5 \\
6-7\end{array}$ & $\begin{array}{l}20-30 \\
50-60\end{array}$ & $\begin{array}{c}5-7 \\
8-10\end{array}$ & $\begin{array}{c}40-70 \\
70-100\end{array}$ & $\begin{array}{c}5-7 \\
8-10\end{array}$ & $\begin{array}{c}70-90 \\
80-120\end{array}$ & $\begin{array}{c}8-9 \\
8-10\end{array}$ & $\begin{array}{l}110-130 \\
120-160\end{array}$ & $\begin{array}{c}8-10 \\
12-14\end{array}$ & $\begin{array}{l}120-140 \\
150-200\end{array}$ \\
\hline 'Santhica 70' & R3 & $01 / 09 / 2019$ & & & & & & & & & & & & $7-8$ & $40-50$ \\
\hline
\end{tabular}


Approximately two weeks following the first sowing (December 12, 2019) the Chinese plots reached the second leaf stage and were generally less uniform with more empty gaps than the French cultivar plots. Northern 'Han FN Q' and southern 'Puma 3', 'Bama' and 'Si-1' germinated quickly and evenly while northern 'Han FN H' and 'Han NW' were particularly slow becoming established (Table 8).

Three weeks later (January 2, 2019) northern cultivars 'Han FN H' and 'Han NW' continued their slow growth. Canopy closure was beginning in the first-sown Chinese plots and the second sowings were reaching the second leaf stage. By January 16, 'Han FN Q' had reached $100-120 \mathrm{~cm}$ at the E site and 80-120 cm at the R site and had begun to flower. By January 22, 'Han NE' had grown to $140-160 \mathrm{~cm}$ at the E site and $110-130 \mathrm{~cm}$ at the R site and was beginning to flower. The remainder of the northern Chinese cultivars commenced flowering by February 6 and at the E plots ranged from $120-170 \mathrm{~cm}$ and at the R plots from $110-200 \mathrm{~cm}$ in height.

Table 8. Crop development stages-Vegetative—Chinese cultivars—Northern biotypes (2018-2019)

\begin{tabular}{|c|c|c|c|c|c|c|c|c|c|c|c|c|c|c|c|}
\hline Observatio & Field & Sowing Date & 12/1222018 & 01/02/2019 & $\begin{array}{c}\text { Crop } \\
\text { Height } \\
(\mathrm{cm}) \\
01 / 0222019\end{array}$ & 01/16/2019 & $\begin{array}{c}\text { Crop } \\
\text { Height } \\
(\mathrm{cm}) \\
01 / 16 / 2019\end{array}$ & 01/22/2019 & $\begin{array}{c}\text { Crop } \\
\text { Height } \\
\text { (cm) } \\
01 / 22 / 2019\end{array}$ & 01/29/2019 & $\begin{array}{c}\text { Crop } \\
\text { Height } \\
\text { (cm) } \\
0129 / 2019\end{array}$ & 02062019 & $\begin{array}{c}\text { Crop } \\
\text { Height } \\
\text { (cm) } \\
02 / 06 / 2019\end{array}$ & 02/13/2019 & $\begin{array}{c}\text { Crop } \\
\text { Height } \\
\text { (om) } \\
02 / 13 / 2019\end{array}$ \\
\hline $\begin{array}{l}\text { 'Han NE' } \\
\text { 'Han NE' }\end{array}$ & $\begin{array}{l}\text { R1 } \\
\text { E1 }\end{array}$ & $\begin{array}{l}11 / 28 / 2018 \\
11 / 29 / 2018\end{array}$ & $\begin{array}{l}2^{\text {nd }} \text { leaf } \\
2^{\text {ndd }} \text { leaf }\end{array}$ & $\begin{array}{c}4-5 \\
5\end{array}$ & $\begin{array}{l}50-60 \\
50-60\end{array}$ & $\begin{array}{l}7-8 \\
7-9\end{array}$ & $\begin{array}{c}90-110 \\
130-140\end{array}$ & $\begin{array}{l}7-9 \\
8-9\end{array}$ & $\begin{array}{l}110-130 \\
140-160\end{array}$ & $\begin{array}{l}8-10 \\
9-10\end{array}$ & $\begin{array}{l}130-150 \\
150-170\end{array}$ & $\begin{array}{c}9-10 \\
10-11\end{array}$ & $\begin{array}{l}150-180 \\
160-190\end{array}$ & $\begin{array}{l}11-12 \\
12-14\end{array}$ & $\begin{array}{l}170-210 \\
200-220\end{array}$ \\
\hline $\begin{array}{l}\text { 'Han NE' } \\
\text { 'Han NE' }\end{array}$ & $\begin{array}{l}\text { R2 } \\
\text { E2 }\end{array}$ & $\begin{array}{l}12 / 22 / 2018 \\
12 / 23 / 2018\end{array}$ & & $\begin{array}{l}2^{\text {nd }} \text { leaf } \\
1^{\text {st }} \text { leaf }\end{array}$ & : & $\begin{array}{l}4-5 \\
5-6\end{array}$ & $\begin{array}{l}20-30 \\
40-50\end{array}$ & $\begin{array}{l}5-6 \\
5-7\end{array}$ & $\begin{array}{l}50-70 \\
60-80\end{array}$ & $\begin{array}{l}5-7 \\
5-7\end{array}$ & $\begin{array}{l}80-100 \\
80-100\end{array}$ & $\begin{array}{l}6-8 \\
6-8\end{array}$ & $\begin{array}{l}100-120 \\
100-120\end{array}$ & $\begin{array}{l}8-10 \\
8-10\end{array}$ & $\begin{array}{l}140-160 \\
120-160\end{array}$ \\
\hline 'Han NE' & R3 & $01 / 09 / 2019$ & & & & & & & & & & & & $5-6$ & $30-40$ \\
\hline $\begin{array}{l}\text { 'Han NW' } \\
\text { 'Han NW' }\end{array}$ & $\begin{array}{l}\text { R1 } \\
\text { E1 }\end{array}$ & $\begin{array}{l}11 / 28 / 2018 \\
11 / 29 / 2018\end{array}$ & $\begin{array}{l}2^{\text {nd }} \text { leaf } \\
2^{\text {nds }} \text { leaf }\end{array}$ & $\begin{array}{l}4-5 \\
4-5\end{array}$ & $\begin{array}{l}60-70 \\
30-40\end{array}$ & $\begin{array}{l}6-7 \\
8-9\end{array}$ & $\begin{array}{c}80-100 \\
100-120\end{array}$ & $\begin{array}{c}7-9 \\
8-10\end{array}$ & $\begin{array}{l}110-130 \\
110-130\end{array}$ & $\begin{array}{l}8-10 \\
9-11\end{array}$ & $\begin{array}{l}130-160 \\
120-160\end{array}$ & $\begin{array}{l}9-11 \\
9-11\end{array}$ & $\begin{array}{l}160-190 \\
140-170\end{array}$ & $\begin{array}{l}12-14 \\
12-14\end{array}$ & $\begin{array}{l}170-220 \\
180-200\end{array}$ \\
\hline $\begin{array}{l}\text { 'Han NW' } \\
\text { 'Han NW' }\end{array}$ & $\begin{array}{l}\mathrm{R} 2 \\
\mathrm{E} 2\end{array}$ & $\begin{array}{l}12 / 22 / 2018 \\
12 / 23 / 2018\end{array}$ & & $\begin{array}{l}2^{\text {nd }} \text { leaf } \\
1^{\text {st }} \text { leaf }\end{array}$ & : & $\begin{array}{l}3-4 \\
4-5\end{array}$ & $\begin{array}{l}20-30 \\
30-40\end{array}$ & $\begin{array}{l}4-5 \\
5-6\end{array}$ & $\begin{array}{l}50-70 \\
60-80\end{array}$ & $\begin{array}{l}5-6 \\
5-7\end{array}$ & $\begin{array}{l}80-100 \\
80-100\end{array}$ & $\begin{array}{l}5-7 \\
6-8\end{array}$ & $\begin{array}{l}100-120 \\
100-120\end{array}$ & $\begin{array}{c}8-9 \\
8-10\end{array}$ & $\begin{array}{l}100-120 \\
100-120\end{array}$ \\
\hline 'Han NW' & R3 & 01/09/2019 & & & & & & & & & & & & $4-5$ & $20-30$ \\
\hline $\begin{array}{l}\text { 'Han Cold' } \\
\text { 'Han Cold' }\end{array}$ & $\begin{array}{l}\text { R1 } \\
\text { E1 }\end{array}$ & $\begin{array}{l}11 / 28 / 2018 \\
11 / 29 / 2018\end{array}$ & $\begin{array}{l}2^{\text {nd }} \text { leaf } \\
2^{\text {ndd }} \text { leaf }\end{array}$ & $\begin{array}{c}4-5 \\
5\end{array}$ & $\begin{array}{l}40-50 \\
50-60\end{array}$ & $\begin{array}{l}6-7 \\
7-8\end{array}$ & $\begin{array}{l}80-100 \\
90-110\end{array}$ & $\begin{array}{l}7-8 \\
7-8\end{array}$ & $\begin{array}{c}90-110 \\
100-120\end{array}$ & $\begin{array}{l}7-9 \\
7-9\end{array}$ & $\begin{array}{l}100-120 \\
110-140\end{array}$ & $\begin{array}{l}8-9 \\
8-9\end{array}$ & $\begin{array}{l}110-140 \\
120-160\end{array}$ & $\begin{array}{c}9-11 \\
10-12\end{array}$ & $\begin{array}{l}140-160 \\
150-180\end{array}$ \\
\hline $\begin{array}{l}\text { 'Han Cold' } \\
\text { 'Han Cold' }\end{array}$ & $\begin{array}{l}\mathrm{R} 2 \\
\mathrm{E} 2\end{array}$ & $\begin{array}{l}12 / 22 / 2018 \\
12 / 23 / 2018\end{array}$ & & $\begin{array}{l}2^{\text {nd }} \text { leaf } \\
1^{\text {st }} \text { leaf }\end{array}$ & : & $\begin{array}{l}5-6 \\
5-6\end{array}$ & $\begin{array}{l}30-40 \\
30-40\end{array}$ & $\begin{array}{l}5-6 \\
6-7\end{array}$ & $\begin{array}{l}40-50 \\
60-80\end{array}$ & $\begin{array}{l}6-7 \\
7-8\end{array}$ & $\begin{array}{c}50-70 \\
80-110\end{array}$ & $\begin{array}{l}6-7 \\
7-9\end{array}$ & $\begin{array}{c}60-80 \\
100-130\end{array}$ & $\begin{array}{c}7-8 \\
10-12\end{array}$ & $\begin{array}{c}90-120 \\
160-220\end{array}$ \\
\hline $\begin{array}{l}\text { 'Han FN H' } \\
\text { 'Han FN H' }\end{array}$ & $\begin{array}{l}\text { R1 } \\
\text { E1 }\end{array}$ & $\begin{array}{l}11 / 28 / 2018 \\
11 / 29 / 2018\end{array}$ & $\begin{array}{l}2^{\text {nd }} \text { leaf } \\
2^{\text {nd }} \text { leaf }\end{array}$ & $\begin{array}{l}6 \\
6\end{array}$ & $\begin{array}{l}50-60 \\
40-50\end{array}$ & $\begin{array}{l}8-9 \\
7-9\end{array}$ & $\begin{array}{c}120-140 \\
90-120\end{array}$ & $\begin{array}{c}8-9 \\
8-10\end{array}$ & $\begin{array}{l}130-150 \\
100-130\end{array}$ & $\begin{array}{l}9-10 \\
9-10\end{array}$ & $\begin{array}{l}150-180 \\
110-150\end{array}$ & $\begin{array}{l}9-10 \\
9-10\end{array}$ & $\begin{array}{l}160-200 \\
130-170\end{array}$ & $\begin{array}{l}11-12 \\
11-12\end{array}$ & $\begin{array}{l}170-220 \\
180-220\end{array}$ \\
\hline $\begin{array}{l}\text { 'Han FN H' } \\
\text { 'Han FN H' }\end{array}$ & $\begin{array}{l}\mathrm{R} 2 \\
\mathrm{E} 2\end{array}$ & $\begin{array}{l}12 / 22 / 2018 \\
12 / 23 / 2018\end{array}$ & & $\begin{array}{l}2^{\text {nd }} \text { leaf } \\
1^{\text {st }} \text { leaf }\end{array}$ & . & $\begin{array}{l}4-5 \\
5-6\end{array}$ & $\begin{array}{l}20-30 \\
30-50\end{array}$ & $\begin{array}{l}4-6 \\
5-6\end{array}$ & $\begin{array}{l}40-60 \\
50-70\end{array}$ & $\begin{array}{l}5-7 \\
5-7\end{array}$ & $\begin{array}{c}70-90 \\
80-110\end{array}$ & $\begin{array}{l}6-7 \\
6-7\end{array}$ & $\begin{array}{l}100-120 \\
100-120\end{array}$ & $\begin{array}{c}8-10 \\
7-8\end{array}$ & $\begin{array}{l}120-160 \\
100-140\end{array}$ \\
\hline 'Han FN H' & R3 & 01/09/2019 & & & & & & & & & & & & $5-6$ & $40-50$ \\
\hline $\begin{array}{l}\text { 'Han FN Q' } \\
\text { 'Han FN Q' }\end{array}$ & $\begin{array}{l}\text { R1 } \\
\text { E1 }\end{array}$ & $\begin{array}{l}11 / 28 / 2018 \\
11 / 29 / 2018\end{array}$ & $\begin{array}{l}2^{\text {ndd }} \text { leaf } \\
2^{\text {ndd }} \text { leaf }\end{array}$ & $\begin{array}{l}5 \\
6\end{array}$ & $\begin{array}{l}40-50 \\
60-70\end{array}$ & $\begin{array}{l}7-8 \\
7-9\end{array}$ & $\begin{array}{c}80-100 \\
100-120\end{array}$ & $\begin{array}{l}7-9 \\
7-9\end{array}$ & $\begin{array}{l}100-120 \\
120-140\end{array}$ & $\begin{array}{l}8-10 \\
9-11\end{array}$ & $\begin{array}{l}120-150 \\
130-150\end{array}$ & $\begin{array}{l}9-10 \\
9-11\end{array}$ & $\begin{array}{l}140-170 \\
140-170\end{array}$ & $\begin{array}{l}10-12 \\
12-14\end{array}$ & $\begin{array}{l}170-220 \\
180-220\end{array}$ \\
\hline $\begin{array}{l}\text { 'Han FN Q' } \\
\text { 'Han FN Q' }\end{array}$ & $\begin{array}{l}\mathrm{R} 2 \\
\mathrm{E} 2\end{array}$ & $\begin{array}{l}12 / 22 / 2018 \\
12 / 23 / 2018\end{array}$ & & $\begin{array}{l}2^{\text {nd }} \text { leaf } \\
1^{\text {st }} \text { leaf }\end{array}$ & : & $\begin{array}{l}4-5 \\
5-6\end{array}$ & $\begin{array}{l}20-30 \\
30-40\end{array}$ & $\begin{array}{l}5-6 \\
5-7\end{array}$ & $\begin{array}{l}50-70 \\
50-80\end{array}$ & $\begin{array}{l}5-7 \\
5-7\end{array}$ & $\begin{array}{l}70-100 \\
70-100\end{array}$ & $\begin{array}{l}6-8 \\
6-8\end{array}$ & $\begin{array}{l}100-120 \\
100-120\end{array}$ & $\begin{array}{l}8-10 \\
8-10\end{array}$ & $\begin{array}{l}120-140 \\
100-140\end{array}$ \\
\hline 'Han FN Q' & R3 & 01/09/2019 & & & & & & & & & & & & $5-6$ & $40-60$ \\
\hline
\end{tabular}

Table 9. Crop development stages—Vegetative—Chinese cultivars—Southern biotypes (2018-2019)

\begin{tabular}{|c|c|c|c|c|c|c|c|c|c|c|c|c|c|c|c|}
\hline Variety & Field & $\begin{array}{c}\text { Sowing } \\
\text { Date }\end{array}$ & 12/12/2018 & 01/022019 & $\begin{array}{c}\text { Crop } \\
\text { Height } \\
(\mathrm{cm}) \\
01 / 022019\end{array}$ & 01/16/2019 & $\begin{array}{c}\text { Crop } \\
\text { Height } \\
\text { (cm) } \\
01 / 1662019\end{array}$ & 01/222019 & $\begin{array}{c}\text { Crop } \\
\text { Height } \\
(\mathrm{cm}) \\
01 / 222019\end{array}$ & 01/29/2019 & $\begin{array}{c}\text { Crop } \\
\text { Height } \\
\text { (cm) } \\
01 / 29 / 2019\end{array}$ & 02/06/2019 & $\begin{array}{c}\text { Crop } \\
\text { Height } \\
\text { (cm) } \\
02 / 06 / 2019\end{array}$ & $\begin{array}{l}\text { Nodes } \\
\text { 02/13/2019 }\end{array}$ & $\begin{array}{c}\text { Crop } \\
\text { Height } \\
\text { (cm) } \\
02 / 13 / 2019\end{array}$ \\
\hline $\begin{array}{l}\text { ‘Bama' } \\
\text { ‘Bama' }\end{array}$ & $\begin{array}{l}\text { R1 } \\
\text { E1 }\end{array}$ & $\begin{array}{l}11 / 28 / 2018 \\
11 / 29 / 2018\end{array}$ & $\begin{array}{l}2^{\text {nd }} \text { leaf } \\
2^{\text {nd }} \text { leaf }\end{array}$ & $\begin{array}{c}4-5 \\
6\end{array}$ & $\begin{array}{l}50-60 \\
40-50\end{array}$ & $\begin{array}{l}8-9 \\
7-8\end{array}$ & $\begin{array}{l}100-120 \\
130-140\end{array}$ & $\begin{array}{c}9-10 \\
8-9\end{array}$ & $\begin{array}{l}120-140 \\
140-150\end{array}$ & $\begin{array}{c}10-11 \\
9-10\end{array}$ & $\begin{array}{l}150-180 \\
150-170\end{array}$ & $\begin{array}{l}10-11 \\
10-12\end{array}$ & $\begin{array}{l}170-210 \\
150-180\end{array}$ & $\begin{array}{l}10-12 \\
12-14\end{array}$ & $\begin{array}{l}180-240 \\
180-220\end{array}$ \\
\hline $\begin{array}{l}\text { 'Bama' } \\
\text { 'Bama' }\end{array}$ & $\begin{array}{l}\text { R2 } \\
\text { E2 }\end{array}$ & $\begin{array}{l}12 / 22 / 2018 \\
12 / 23 / 2018\end{array}$ & & $\begin{array}{l}2^{\text {nd }} \text { leaf } \\
1^{\text {st }} \text { leaf }\end{array}$ & $\cdot$ & $\begin{array}{l}3-4 \\
5-6\end{array}$ & $\begin{array}{l}20-30 \\
20-40\end{array}$ & $\begin{array}{l}5-6 \\
5-7\end{array}$ & $\begin{array}{l}40-60 \\
40-60\end{array}$ & $\begin{array}{l}6-7 \\
5-7\end{array}$ & $\begin{array}{l}70-90 \\
70-90\end{array}$ & $\begin{array}{l}6-8 \\
6-8\end{array}$ & $\begin{array}{l}100-120 \\
100-120\end{array}$ & $\begin{array}{l}8-10 \\
8-10\end{array}$ & $\begin{array}{l}120-140 \\
100-160\end{array}$ \\
\hline 'Bama' & R3 & 01/09/2019 & & & & & & & & & & & & $5-7$ & $50-60$ \\
\hline $\begin{array}{l}\text { 'Puma 3' } \\
\text { 'Puma 3' }\end{array}$ & $\begin{array}{l}\text { R1 } \\
\text { E1 }\end{array}$ & $\begin{array}{l}11 / 28 / 2018 \\
11 / 29 / 2018\end{array}$ & $\begin{array}{l}2^{\text {nd }} \text { leaf } \\
2^{\text {nd }} \text { leaf }\end{array}$ & $\begin{array}{l}5 \\
6\end{array}$ & $\begin{array}{l}30-50 \\
60-70\end{array}$ & $\begin{array}{l}6-7 \\
7-8\end{array}$ & $\begin{array}{l}100-120 \\
100-120\end{array}$ & $\begin{array}{l}6-7 \\
7-9\end{array}$ & $\begin{array}{l}110-120 \\
110-130\end{array}$ & $\begin{array}{l}6-8 \\
8-9\end{array}$ & $\begin{array}{l}120-130 \\
120-140\end{array}$ & $\begin{array}{c}7-8 \\
8-10\end{array}$ & $\begin{array}{l}120-140 \\
140-170\end{array}$ & $\begin{array}{c}8-10 \\
11-12\end{array}$ & $\begin{array}{l}140-160 \\
180-200\end{array}$ \\
\hline $\begin{array}{l}\text { 'Puma 3' } \\
\text { 'Puma 3' }\end{array}$ & $\begin{array}{l}\text { R2 } \\
\text { E2 }\end{array}$ & $\begin{array}{l}12 / 22 / 2018 \\
12 / 23 / 2018\end{array}$ & & $\begin{array}{l}2^{\text {nd }} \text { leaf } \\
1^{\text {st }} \text { leaf }\end{array}$ & $\dot{-}$ & $\begin{array}{l}3-4 \\
5-6\end{array}$ & $\begin{array}{l}20-30 \\
30-40\end{array}$ & $\begin{array}{l}4-6 \\
5-7\end{array}$ & $\begin{array}{l}30-50 \\
40-50\end{array}$ & $\begin{array}{l}5-7 \\
6-7\end{array}$ & $\begin{array}{l}60-80 \\
60-80\end{array}$ & $\begin{array}{l}7-8 \\
6-7\end{array}$ & $\begin{array}{l}80-100 \\
90-110\end{array}$ & $\begin{array}{c}8-10 \\
6-8\end{array}$ & $\begin{array}{l}140-160 \\
100-120\end{array}$ \\
\hline 'Puma 3' & R3 & 01/09/2019 & & & & & & & & & & & & $5-7$ & $40-60$ \\
\hline $\begin{array}{l}\text { 'Yuma' } \\
\text { 'Yuma' }\end{array}$ & $\begin{array}{l}\text { R1 } \\
\text { E1 }\end{array}$ & $\begin{array}{l}11 / 28 / 2018 \\
11 / 29 / 2018\end{array}$ & $\begin{array}{l}2^{\text {nd }} \text { leaf } \\
2^{\text {nd }} \text { leaf }\end{array}$ & $\begin{array}{l}6 \\
6\end{array}$ & $\begin{array}{l}70-80 \\
40-50\end{array}$ & $\begin{array}{l}7-8 \\
7-8\end{array}$ & $\begin{array}{c}90-110 \\
100-120\end{array}$ & $\begin{array}{l}8-9 \\
8-9\end{array}$ & $\begin{array}{l}120-160 \\
120-140\end{array}$ & $\begin{array}{l}9-10 \\
9-10\end{array}$ & $\begin{array}{l}150-180 \\
130-160\end{array}$ & $\begin{array}{l}9-11 \\
9-11\end{array}$ & $\begin{array}{l}170-210 \\
150-210\end{array}$ & $\begin{array}{l}11-12 \\
11-12\end{array}$ & $\begin{array}{l}180-220 \\
180-200\end{array}$ \\
\hline $\begin{array}{l}\text { 'Yuma' } \\
\text { 'Yuma' }\end{array}$ & $\begin{array}{l}\text { R2 } \\
\text { E2 }\end{array}$ & $\begin{array}{l}12 / 22 / 2018 \\
12 / 23 / 2018\end{array}$ & & $\begin{array}{l}2^{\text {nd }} \text { leaf } \\
1^{\text {st }} \text { leaf }\end{array}$ & $\cdot$ & $\begin{array}{l}4-5 \\
5-6\end{array}$ & $\begin{array}{l}20-30 \\
20-30\end{array}$ & $\begin{array}{l}5-6 \\
5-7\end{array}$ & $\begin{array}{l}50-70 \\
60-70\end{array}$ & $\begin{array}{l}5-7 \\
6-7\end{array}$ & $\begin{array}{l}80-100 \\
80-100\end{array}$ & $\begin{array}{l}6-8 \\
6-7\end{array}$ & $\begin{array}{l}110-130 \\
100-120\end{array}$ & $\begin{array}{c}8-10 \\
7-8\end{array}$ & $\begin{array}{l}140-160 \\
120-160\end{array}$ \\
\hline 'Yuma' & R3 & $01 / 09 / 2019$ & & & & & & & & & & & & $6-7$ & $10-40$ \\
\hline $\begin{array}{l}\text { 'Si-1' } \\
\text { 'Si-1' }\end{array}$ & $\begin{array}{l}\text { R1 } \\
\text { E1 }\end{array}$ & $\begin{array}{l}11 / 28 / 2018 \\
11 / 29 / 2018\end{array}$ & $\begin{array}{l}2^{\text {nd }} \text { leaf } \\
2^{\text {nd }} \text { leaf }\end{array}$ & $\begin{array}{l}5 \\
5\end{array}$ & $\begin{array}{l}40-50 \\
50-60\end{array}$ & $\begin{array}{l}7-8 \\
7-8\end{array}$ & $\begin{array}{l}90-110 \\
90-110\end{array}$ & $\begin{array}{l}8-9 \\
8-9\end{array}$ & $\begin{array}{l}110-130 \\
110-130\end{array}$ & $\begin{array}{l}9-10 \\
9-10\end{array}$ & $\begin{array}{l}120-150 \\
120-140\end{array}$ & $\begin{array}{l}9-10 \\
9-10\end{array}$ & $\begin{array}{l}130-170 \\
140-160\end{array}$ & $\begin{array}{l}10-12 \\
10-12\end{array}$ & $\begin{array}{l}170-200 \\
180-200\end{array}$ \\
\hline $\begin{array}{l}\text { 'Si-1' } \\
\text { 'Si-1' }\end{array}$ & $\begin{array}{l}\text { R2 } \\
\text { E2 }\end{array}$ & $\begin{array}{l}12 / 22 / 2018 \\
12 / 23 / 2018\end{array}$ & & $\begin{array}{l}2^{\text {nd }} \text { leaf } \\
1^{\text {st }} \text { leaf }\end{array}$ & : & $\begin{array}{l}4-5 \\
5-6\end{array}$ & $\begin{array}{l}20-30 \\
40-60\end{array}$ & $\begin{array}{l}4-6 \\
5-7\end{array}$ & $\begin{array}{l}50-60 \\
60-80\end{array}$ & $\begin{array}{l}5-7 \\
6-8\end{array}$ & $\begin{array}{l}80-100 \\
80-110\end{array}$ & $\begin{array}{l}5-7 \\
6-8\end{array}$ & $\begin{array}{l}100-120 \\
100-140\end{array}$ & $\begin{array}{c}8-9 \\
8-10\end{array}$ & $\begin{array}{l}130-150 \\
140-160\end{array}$ \\
\hline 'Si-1' & R3 & 01/09/2019 & & & & & & & & & & & & $4-5$ & $30-50$ \\
\hline
\end{tabular}

By January 29, the southern Chinese cultivars 'Bama', 'Puma 3'and 'Yuma' had begun to flower. 'Puma 3' reached 120-140 cm at the E site and 120-130 cm at the R site while 'Bama' and 'Yuma' reached 130-160 cm at the E site and $150-180 \mathrm{~cm}$ at the R site. 'Si-1' reached $140-170 \mathrm{~cm}$ at the $\mathrm{E}$ site and $130-180 \mathrm{~cm}$ at the R site as it began to flower. In 
the second sowing, 'Bama' and 'Yuma' had begun flowering by February 6 having reached 100-120 cm in height at the E site and 100-130 cm at the R site. 'Puma' had reached 100-120 cm at the E site and 140-160 cm at the R site on February 13 and had begun flowering. By the end of March the second sowing of 'Si-1' was yet to flower (Table 9).

\subsection{Crop growth and development—Flowering stages}

The wide range of latitudes represented by the cultivars to be trialed led us to expect a wide range of flowering responses. By January 16, 2019, male flowers were beginning to appear in the first sowing of all four French cultivars especially in the few (approx. ten percent) of off-type purely staminate male plants within the generally monoecious population. Later as the seeds ripened pure pistillate female plants were also observed at similar levels. The second sowing of French cultivars had all begun flowering by January 29. By February 13, seed was set in all of the first-sown plots and in the second-sown plots only 'Ferimon' began to flower earlier at the E trial site. Seeds were ripening well in all French cultivars across all trial plots by the first week of April (Table 10). The third sowing of French cultivars neither flowered nor set seed before the trials ended.

The Canadian cultivars commenced male and female flowering by January 2 and seeds were beginning to mature in early February. 'CFX-1' and 'Picolo' began to set seed by January 29 and 'CRS-1' and 'Katani' by February 6. All four Canadian cultivars were ripe and awaiting harvest by mid-March (Table 10).

Table 10. Crop development stages—Flowering—Canadian and French cultivars (2018-2019)

\begin{tabular}{|c|c|c|c|c|c|c|c|c|c|c|c|c|}
\hline \multirow{2}{*}{$\begin{array}{l}\text { Variety } \\
\text { Observation Date }\end{array}$} & \multirow[t]{2}{*}{ Field } & \multirow[t]{2}{*}{$\begin{array}{l}\text { Sowing } \\
\text { Date }\end{array}$} & \multicolumn{6}{|c|}{$\begin{array}{c}\begin{array}{c}\text { Flowering } \\
\text { (Male/Female) }\end{array} \\
\text { (0=no floral initiation; 1 =floral initiation; } 2=\text { =inflorescence development; 3=full flowering) }\end{array}$} & \multicolumn{4}{|c|}{$\begin{array}{c}\text { Seed Set } \\
(\mathrm{Yes} / \mathrm{No})\end{array}$} \\
\hline & & & $01 / 02 / 2019$ & 01/16/2019 & 01/22/2019 & 01/29/2019 & 02/06/2019 & $02 / 13 / 2019$ & 01/22/2019 & 01/29/2019 & $02 / 06 / 2019$ & $02 / 13 / 2019$ \\
\hline $\begin{array}{l}\text { 'CFX-1' } \\
\text { 'CFX-1' }\end{array}$ & $\begin{array}{l}\text { R2 } \\
\text { E2 }\end{array}$ & $\begin{array}{l}12 / 22 / 2018 \\
12 / 23 / 2018\end{array}$ & $\begin{array}{l}1 / 1 \\
1 / 1\end{array}$ & $\begin{array}{l}1 / 1 \\
1 / 1\end{array}$ & $\begin{array}{l}2 / 2 \\
2 / 2\end{array}$ & $\begin{array}{l}2 / 2 \\
2 / 2\end{array}$ & $\begin{array}{l}2 / 2 \\
2 / 2\end{array}$ & $\begin{array}{l}3 / 2 \\
3 / 2\end{array}$ & $\begin{array}{l}\text { No } \\
\text { No }\end{array}$ & $\begin{array}{l}\text { Yes } \\
\text { Yes }\end{array}$ & $\begin{array}{l}\text { Yes } \\
\text { Yes }\end{array}$ & $\begin{array}{l}\text { Yes } \\
\text { Yes }\end{array}$ \\
\hline $\begin{array}{l}\text { ‘CRS-1' } \\
\text { 'CRS-1' }\end{array}$ & $\begin{array}{l}\text { R2 } \\
\text { E2 }\end{array}$ & $\begin{array}{l}12 / 22 / 2018 \\
12 / 23 / 2018\end{array}$ & $\begin{array}{l}1 / 1 \\
1 / 1\end{array}$ & $\begin{array}{l}1 / 1 \\
1 / 1\end{array}$ & $\begin{array}{l}2 / 2 \\
2 / 2\end{array}$ & $\begin{array}{l}2 / 2 \\
2 / 2\end{array}$ & $\begin{array}{l}2 / 2 \\
2 / 2\end{array}$ & $\begin{array}{l}3 / 2 \\
3 / 2\end{array}$ & $\begin{array}{l}\text { No } \\
\text { No }\end{array}$ & $\begin{array}{l}\text { No } \\
\text { No }\end{array}$ & $\begin{array}{l}\text { Yes } \\
\text { Yes }\end{array}$ & $\begin{array}{l}\text { Yes } \\
\text { Yes }\end{array}$ \\
\hline $\begin{array}{l}\text { 'Katani' } \\
\text { 'Katani' }\end{array}$ & $\begin{array}{l}\text { R2 } \\
\text { E2 }\end{array}$ & $\begin{array}{l}12 / 22 / 2018 \\
12 / 23 / 2018\end{array}$ & $\begin{array}{l}1 / 1 \\
1 / 1\end{array}$ & $\begin{array}{l}1 / 1 \\
1 / 1\end{array}$ & $\begin{array}{l}2 / 2 \\
2 / 2\end{array}$ & $\begin{array}{l}2 / 2 \\
2 / 2\end{array}$ & $\begin{array}{l}2 / 2 \\
2 / 2\end{array}$ & $\begin{array}{l}3 / 2 \\
3 / 2\end{array}$ & $\begin{array}{l}\text { No } \\
\text { No }\end{array}$ & $\begin{array}{l}\text { No } \\
\text { No }\end{array}$ & $\begin{array}{l}\text { Yes } \\
\text { Yes }\end{array}$ & $\begin{array}{l}\text { Yes } \\
\text { Yes }\end{array}$ \\
\hline $\begin{array}{l}\text { 'Picolo' } \\
\text { 'Picolo' }\end{array}$ & $\begin{array}{l}\text { R2 } \\
\text { E2 }\end{array}$ & $\begin{array}{l}12 / 22 / 2018 \\
12 / 23 / 2018\end{array}$ & $\begin{array}{l}1 / 1 \\
1 / 1\end{array}$ & $\begin{array}{l}1 / 1 \\
1 / 1\end{array}$ & $\begin{array}{l}2 / 2 \\
2 / 2\end{array}$ & $\begin{array}{l}2 / 2 \\
2 / 2\end{array}$ & $\begin{array}{l}2 / 2 \\
2 / 2\end{array}$ & $\begin{array}{l}3 / 2 \\
3 / 2\end{array}$ & $\begin{array}{l}\text { No } \\
\text { No }\end{array}$ & $\begin{array}{l}\text { Yes } \\
\text { Yes }\end{array}$ & $\begin{array}{l}\text { Yes } \\
\text { Yes }\end{array}$ & $\begin{array}{l}\text { Yes } \\
\text { Yes }\end{array}$ \\
\hline $\begin{array}{l}\text { 'Felina 32' } \\
\text { 'Felina 32' }\end{array}$ & $\begin{array}{l}\text { R1 } \\
\text { E1 }\end{array}$ & $\begin{array}{l}11 / 28 / 2018 \\
11 / 29 / 2018\end{array}$ & $\begin{array}{l}0 / 0 \\
0 / 0\end{array}$ & $\begin{array}{l}1 / 1 \\
1 / 1\end{array}$ & $\begin{array}{l}2 / 2 \\
2 / 2\end{array}$ & $\begin{array}{l}2 / 2 \\
2 / 2\end{array}$ & $\begin{array}{l}2 / 2 \\
2 / 2\end{array}$ & $\begin{array}{l}3 / 2 \\
2 / 2\end{array}$ & $\begin{array}{l}\text { No } \\
\text { No }\end{array}$ & $\begin{array}{l}\text { No } \\
\text { No }\end{array}$ & $\begin{array}{l}\text { No } \\
\text { Yes }\end{array}$ & $\begin{array}{l}\text { Yes } \\
\text { Yes }\end{array}$ \\
\hline $\begin{array}{l}\text { ‘Felina 32' } \\
\text { 'Felina 32' }\end{array}$ & $\begin{array}{l}\text { R2 } \\
\text { E2 }\end{array}$ & $\begin{array}{l}12 / 22 / 2018 \\
12 / 23 / 2018\end{array}$ & $\begin{array}{l}0 / 0 \\
0 / 0\end{array}$ & $\begin{array}{l}0 / 0 \\
0 / 0\end{array}$ & $\begin{array}{l}0 / 0 \\
0 / 0\end{array}$ & $\begin{array}{l}1 / 1 \\
1 / 1\end{array}$ & $\begin{array}{l}1 / 1 \\
2 / 2\end{array}$ & $\begin{array}{l}2 / 1 \\
2 / 2\end{array}$ & $\begin{array}{l}\text { No } \\
\text { No }\end{array}$ & $\begin{array}{l}\text { No } \\
\text { No }\end{array}$ & $\begin{array}{l}\text { No } \\
\text { No }\end{array}$ & $\begin{array}{l}\text { No } \\
\text { No }\end{array}$ \\
\hline 'Felina 32' & R3 & $01 / 09 / 2019$ & $\cdot$ & $0 / 0$ & $0 / 0$ & $0 / 0$ & $0 / 0$ & $0 / 0$ & No & No & No & No \\
\hline $\begin{array}{l}\text { 'Ferimon' } \\
\text { 'Ferimon' }\end{array}$ & $\begin{array}{l}\text { R1 } \\
\text { E1 }\end{array}$ & $\begin{array}{l}11 / 28 / 2018 \\
11 / 29 / 2018\end{array}$ & $\begin{array}{l}0 / 0 \\
0 / 0\end{array}$ & $\begin{array}{l}1 / 1 \\
1 / 1\end{array}$ & $\begin{array}{l}2 / 2 \\
2 / 2\end{array}$ & $\begin{array}{l}2 / 2 \\
2 / 2\end{array}$ & $\begin{array}{l}2 / 2 \\
2 / 2\end{array}$ & $\begin{array}{l}3 / 2 \\
2 / 2\end{array}$ & $\begin{array}{l}\text { No } \\
\text { No }\end{array}$ & $\begin{array}{l}\text { No } \\
\text { No }\end{array}$ & $\begin{array}{l}\text { Yes } \\
\text { Yes }\end{array}$ & $\begin{array}{l}\text { Yes } \\
\text { Yes }\end{array}$ \\
\hline $\begin{array}{l}\text { 'Ferimon' } \\
\text { 'Ferimon' }\end{array}$ & $\begin{array}{l}\text { R2 } \\
\text { E2 }\end{array}$ & $\begin{array}{l}12 / 22 / 2018 \\
12 / 23 / 2018\end{array}$ & $\begin{array}{l}0 / 0 \\
0 / 0\end{array}$ & $\begin{array}{l}0 / 0 \\
0 / 0\end{array}$ & $\begin{array}{l}0 / 0 \\
0 / 0\end{array}$ & $\begin{array}{l}1 / 1 \\
1 / 1\end{array}$ & $\begin{array}{l}2 / 2 \\
2 / 2\end{array}$ & $\begin{array}{l}2 / 2 \\
2 / 2\end{array}$ & $\begin{array}{l}\text { No } \\
\text { No }\end{array}$ & $\begin{array}{l}\text { No } \\
\text { No }\end{array}$ & $\begin{array}{l}\text { No } \\
\text { Yes }\end{array}$ & $\begin{array}{l}\text { No } \\
\text { Yes }\end{array}$ \\
\hline 'Ferimon' & R3 & $01 / 09 / 2019$ & - & $0 / 0$ & $0 / 0$ & $0 / 0$ & $0 / 0$ & $0 / 0$ & No & No & No & No \\
\hline $\begin{array}{l}\text { 'Futura 75' } \\
\text { 'Futura 75' }\end{array}$ & $\begin{array}{l}\text { R1 } \\
\text { E1 }\end{array}$ & $\begin{array}{l}11 / 28 / 2018 \\
11 / 29 / 2018\end{array}$ & $\begin{array}{l}0 / 0 \\
0 / 0\end{array}$ & $\begin{array}{l}1 / 1 \\
1 / 1\end{array}$ & $\begin{array}{l}2 / 1 \\
2 / 2\end{array}$ & $\begin{array}{l}2 / 2 \\
2 / 2\end{array}$ & $\begin{array}{l}2 / 2 \\
2 / 2\end{array}$ & $\begin{array}{l}3 / 2 \\
2 / 2\end{array}$ & $\begin{array}{l}\text { No } \\
\text { No }\end{array}$ & $\begin{array}{l}\text { No } \\
\text { No }\end{array}$ & $\begin{array}{l}\text { No } \\
\text { No }\end{array}$ & $\begin{array}{l}\text { Yes } \\
\text { Yes }\end{array}$ \\
\hline $\begin{array}{l}\text { 'Futura 75' } \\
\text { 'Futura 75' }\end{array}$ & $\begin{array}{l}\text { R2 } \\
\text { E2 }\end{array}$ & $\begin{array}{l}12 / 22 / 2018 \\
12 / 23 / 2018\end{array}$ & $\begin{array}{l}0 / 0 \\
0 / 0\end{array}$ & $\begin{array}{l}0 / 0 \\
0 / 0\end{array}$ & $\begin{array}{l}0 / 0 \\
0 / 0\end{array}$ & $\begin{array}{l}1 / 1 \\
1 / 1\end{array}$ & $\begin{array}{l}2 / 2 \\
2 / 2\end{array}$ & $\begin{array}{l}2 / 2 \\
2 / 2\end{array}$ & $\begin{array}{l}\text { No } \\
\text { No }\end{array}$ & $\begin{array}{l}\text { No } \\
\text { No }\end{array}$ & $\begin{array}{l}\text { No } \\
\text { No }\end{array}$ & $\begin{array}{l}\text { No } \\
\text { No }\end{array}$ \\
\hline 'Futura 75' & R3 & $01 / 09 / 2019$ & $\cdot$ & $0 / 0$ & $0 / 0$ & $0 / 0$ & $0 / 0$ & $0 / 0$ & No & No & No & No \\
\hline $\begin{array}{l}\text { 'Santhica 70' } \\
\text { 'Santhica 70' }\end{array}$ & $\begin{array}{l}\text { R1 } \\
\text { E1 }\end{array}$ & $\begin{array}{l}11 / 28 / 2018 \\
11 / 29 / 2018\end{array}$ & $\begin{array}{l}0 / 0 \\
0 / 0\end{array}$ & $\begin{array}{l}1 / 1 \\
1 / 1\end{array}$ & $\begin{array}{l}2 / 2 \\
2 / 2\end{array}$ & $\begin{array}{l}2 / 2 \\
2 / 2\end{array}$ & $\begin{array}{l}2 / 2 \\
2 / 2\end{array}$ & $\begin{array}{l}3 / 2 \\
3 / 2\end{array}$ & $\begin{array}{l}\text { No } \\
\text { No }\end{array}$ & $\begin{array}{l}\text { No } \\
\text { No }\end{array}$ & $\begin{array}{l}\text { No } \\
\text { Yes }\end{array}$ & $\begin{array}{l}\text { Yes } \\
\text { Yes }\end{array}$ \\
\hline $\begin{array}{l}\text { 'Santhica 70' } \\
\text { 'Santhica 70' }\end{array}$ & $\begin{array}{l}\text { R2 } \\
\text { E2 }\end{array}$ & $\begin{array}{l}12 / 22 / 2018 \\
12 / 23 / 2018\end{array}$ & $\begin{array}{l}0 / 0 \\
0 / 0\end{array}$ & $\begin{array}{l}0 / 0 \\
0 / 0\end{array}$ & $\begin{array}{l}0 / 0 \\
0 / 0\end{array}$ & $\begin{array}{l}1 / 1 \\
1 / 1\end{array}$ & $\begin{array}{l}2 / 2 \\
2 / 2\end{array}$ & $\begin{array}{l}2 / 2 \\
2 / 2\end{array}$ & $\begin{array}{l}\text { No } \\
\text { No }\end{array}$ & $\begin{array}{l}\text { No } \\
\text { No }\end{array}$ & $\begin{array}{l}\text { No } \\
\text { No }\end{array}$ & $\begin{array}{l}\text { No } \\
\text { No }\end{array}$ \\
\hline 'Santhica 70' & R3 & $01 / 09 / 2019$ & - & $0 / 0$ & $0 / 0$ & $0 / 0$ & $0 / 0$ & $0 / 0$ & No & No & No & No \\
\hline
\end{tabular}

All of the Chinese cultivars flowered later than the Canadian and French cultivars. In the first sowing of northern varieties, 'Han FN Q' initiated flowering shortly before January 16 and 'Han NE' was flowering by January 22. 'Han NW' and 'Han FN H' began flowering by February 6 and "Han Cold" failed to flower before the trials were terminated (Table 11). The southern varieties 'Bama', 'Puma 3' and 'Yuma' began flowering prior to January 29 while 'Si-1' began flowering by February 6. In the second sowings, 'Bama' and 'Yuma' commenced flowering a week after the first sowings, 'Puma 3' two weeks later and 'Han FN Q' failed to flower (Table 12). None of the Chinese cultivars set seed. 
Table 11. Crop development stages-Flowering - Chinese cultivars-Northern biotypes (2018-2019)

\begin{tabular}{|c|c|c|c|c|c|c|c|c|c|c|c|c|}
\hline \multirow{2}{*}{\multicolumn{2}{|c|}{$\begin{array}{l}\text { Variety Field } \\
\text { Observation Date }\end{array}$}} & \multirow[t]{2}{*}{$\begin{array}{l}\text { Sowing } \\
\text { Date }\end{array}$} & \multicolumn{6}{|c|}{$\begin{array}{c}\begin{array}{c}\text { Flowering } \\
\text { (Male/Female) }\end{array} \\
(0=\text { no floral initiation; } 1 \text { =floral initiation; } 2=\text { =inflorescence development; } 3=\text { full flowering })\end{array}$} & \multicolumn{4}{|c|}{$\begin{array}{l}\text { Seed Set } \\
\text { (Yes/No) }\end{array}$} \\
\hline & & & $01 / 02 / 2019$ & $01 / 16 / 2019$ & $01 / 22 / 2019$ & $01 / 29 / 2019$ & $02 / 06 / 2019$ & $02 / 13 / 2019$ & $01 / 22 / 2019$ & 01/29/2019 & $02 / 06 / 2019$ & $02 / 13 / 2019$ \\
\hline $\begin{array}{l}\text { 'Han NE' } \\
\text { 'Han NE' }\end{array}$ & $\begin{array}{l}\text { R1 } \\
\text { E1 }\end{array}$ & $\begin{array}{l}11 / 28 / 2018 \\
11 / 29 / 2018\end{array}$ & $\begin{array}{l}0 / 0 \\
0 / 0\end{array}$ & $\begin{array}{l}0 / 0 \\
0 / 0\end{array}$ & $\begin{array}{l}0 / 0 \\
1 / 0\end{array}$ & $\begin{array}{l}1 / 0 \\
1 / 0\end{array}$ & $\begin{array}{l}1 / 0 \\
1 / 0\end{array}$ & $\begin{array}{l}2 / 1 \\
2 / 1\end{array}$ & $\begin{array}{l}\text { No } \\
\text { No }\end{array}$ & $\begin{array}{l}\text { No } \\
\text { No }\end{array}$ & $\begin{array}{l}\text { No } \\
\text { No }\end{array}$ & $\begin{array}{l}\text { No } \\
\text { No }\end{array}$ \\
\hline $\begin{array}{l}\text { 'Han NE' } \\
\text { 'Han NE' }\end{array}$ & $\begin{array}{l}\text { R2 } \\
\text { E2 }\end{array}$ & $\begin{array}{l}12 / 22 / 2018 \\
12 / 23 / 2018\end{array}$ & $\begin{array}{l}0 / 0 \\
0 / 0\end{array}$ & $\begin{array}{l}0 / 0 \\
0 / 0\end{array}$ & $\begin{array}{l}0 / 0 \\
0 / 0\end{array}$ & $\begin{array}{l}1 / 0 \\
1 / 0\end{array}$ & $\begin{array}{l}1 / 0 \\
1 / 0\end{array}$ & $\begin{array}{l}2 / 1 \\
2 / 1\end{array}$ & $\begin{array}{l}\text { No } \\
\text { No }\end{array}$ & $\begin{array}{l}\text { No } \\
\text { No }\end{array}$ & $\begin{array}{l}\text { No } \\
\text { No }\end{array}$ & $\begin{array}{l}\text { No } \\
\text { No }\end{array}$ \\
\hline ‘Han NE' & R3 & $01 / 09 / 2019$ & - & $0 / 0$ & $0 / 0$ & $0 / 0$ & $0 / 0$ & $0 / 0$ & No & No & No & No \\
\hline $\begin{array}{l}\text { 'Han NW' } \\
\text { 'Han NW' }\end{array}$ & $\begin{array}{l}\text { R1 } \\
\text { E1 }\end{array}$ & $\begin{array}{l}11 / 28 / 2018 \\
11 / 29 / 2018\end{array}$ & $\begin{array}{l}0 / 0 \\
0 / 0\end{array}$ & $\begin{array}{l}0 / 0 \\
0 / 0\end{array}$ & $\begin{array}{l}0 / 0 \\
0 / 0\end{array}$ & $\begin{array}{l}0 / 0 \\
0 / 0\end{array}$ & $\begin{array}{l}1 / 0 \\
1 / 0\end{array}$ & $\begin{array}{l}2 / 0 \\
2 / 0\end{array}$ & $\begin{array}{l}\text { No } \\
\text { No }\end{array}$ & $\begin{array}{l}\text { No } \\
\text { No }\end{array}$ & $\begin{array}{l}\text { No } \\
\text { No }\end{array}$ & $\begin{array}{l}\text { No } \\
\text { No }\end{array}$ \\
\hline $\begin{array}{l}\text { 'Han NW' } \\
\text { 'Han NW' }\end{array}$ & $\begin{array}{l}\mathrm{R} 2 \\
\mathrm{E} 2\end{array}$ & $\begin{array}{l}12 / 22 / 2018 \\
12 / 23 / 2018\end{array}$ & $\begin{array}{l}0 / 0 \\
0 / 0\end{array}$ & $\begin{array}{l}0 / 0 \\
0 / 0\end{array}$ & $\begin{array}{l}0 / 0 \\
0 / 0\end{array}$ & $\begin{array}{l}0 / 0 \\
0 / 0\end{array}$ & $\begin{array}{l}1 / 0 \\
1 / 0\end{array}$ & $\begin{array}{l}2 / 1 \\
2 / 1\end{array}$ & $\begin{array}{l}\text { No } \\
\text { No }\end{array}$ & $\begin{array}{l}\text { No } \\
\text { No }\end{array}$ & $\begin{array}{l}\text { No } \\
\text { No }\end{array}$ & $\begin{array}{l}\text { No } \\
\text { No }\end{array}$ \\
\hline 'Han NW' & R3 & $01 / 09 / 2019$ & - & $0 / 0$ & $0 / 0$ & $0 / 0$ & $0 / 0$ & $0 / 0$ & No & No & No & No \\
\hline $\begin{array}{l}\text { 'Han Cold' } \\
\text { 'Han Cold' }\end{array}$ & $\begin{array}{l}\text { R1 } \\
\text { E1 }\end{array}$ & $\begin{array}{l}11 / 28 / 2018 \\
11 / 29 / 2018\end{array}$ & $\begin{array}{l}0 / 0 \\
0 / 0\end{array}$ & $\begin{array}{l}0 / 0 \\
0 / 0\end{array}$ & $\begin{array}{l}0 / 0 \\
0 / 0\end{array}$ & $\begin{array}{l}0 / 0 \\
0 / 0\end{array}$ & $\begin{array}{l}0 / 0 \\
0 / 0\end{array}$ & $\begin{array}{l}0 / 0 \\
0 / 0\end{array}$ & $\begin{array}{l}\text { No } \\
\text { No }\end{array}$ & $\begin{array}{l}\text { No } \\
\text { No }\end{array}$ & $\begin{array}{l}\text { No } \\
\text { No }\end{array}$ & $\begin{array}{l}\text { No } \\
\text { No }\end{array}$ \\
\hline $\begin{array}{l}\text { 'Han Cold' } \\
\text { 'Han Cold' }\end{array}$ & $\begin{array}{l}\text { R2 } \\
\text { E2 }\end{array}$ & $\begin{array}{l}12 / 22 / 2018 \\
12 / 23 / 2018\end{array}$ & $\begin{array}{l}0 / 0 \\
0 / 0\end{array}$ & $\begin{array}{l}0 / 0 \\
0 / 0\end{array}$ & $\begin{array}{l}0 / 0 \\
0 / 0\end{array}$ & $\begin{array}{l}0 / 0 \\
0 / 0\end{array}$ & $\begin{array}{l}0 / 0 \\
0 / 0\end{array}$ & $\begin{array}{l}0 / 0 \\
0 / 0\end{array}$ & $\begin{array}{l}\text { No } \\
\text { No }\end{array}$ & $\begin{array}{l}\text { No } \\
\text { No }\end{array}$ & $\begin{array}{l}\text { No } \\
\text { No }\end{array}$ & $\begin{array}{l}\text { No } \\
\text { No }\end{array}$ \\
\hline $\begin{array}{l}\text { 'Han FN H' } \\
\text { 'Han FN H' }\end{array}$ & $\begin{array}{l}\text { R1 } \\
\text { E1 }\end{array}$ & $\begin{array}{l}11 / 28 / 2018 \\
11 / 29 / 2018\end{array}$ & $\begin{array}{l}0 / 0 \\
0 / 0\end{array}$ & $\begin{array}{l}0 / 0 \\
0 / 0\end{array}$ & $\begin{array}{l}0 / 0 \\
0 / 0\end{array}$ & $\begin{array}{l}0 / 0 \\
0 / 0\end{array}$ & $\begin{array}{l}1 / 0 \\
1 / 0\end{array}$ & $\begin{array}{l}2 / 1 \\
2 / 0\end{array}$ & $\begin{array}{l}\text { No } \\
\text { No }\end{array}$ & $\begin{array}{l}\text { No } \\
\text { No }\end{array}$ & $\begin{array}{l}\text { No } \\
\text { No }\end{array}$ & $\begin{array}{l}\text { No } \\
\text { No }\end{array}$ \\
\hline $\begin{array}{l}\text { 'Han FN H' } \\
\text { 'Han FN H' }\end{array}$ & $\begin{array}{l}\text { R2 } \\
\text { E2 }\end{array}$ & $\begin{array}{l}12 / 22 / 2018 \\
12 / 23 / 2018\end{array}$ & $\begin{array}{l}0 / 0 \\
0 / 0\end{array}$ & $\begin{array}{l}0 / 0 \\
0 / 0\end{array}$ & $\begin{array}{l}0 / 0 \\
0 / 0\end{array}$ & $\begin{array}{l}0 / 0 \\
0 / 0\end{array}$ & $\begin{array}{l}0 / 0 \\
0 / 0\end{array}$ & $\begin{array}{l}1 / 0 \\
1 / 0\end{array}$ & $\begin{array}{l}\text { No } \\
\text { No }\end{array}$ & $\begin{array}{l}\text { No } \\
\text { No }\end{array}$ & $\begin{array}{l}\text { No } \\
\text { No }\end{array}$ & $\begin{array}{l}\text { No } \\
\text { No }\end{array}$ \\
\hline 'Han FN H' & R3 & 01/09/2019 & - & $0 / 0$ & $0 / 0$ & $0 / 0$ & $0 / 0$ & $0 / 0$ & No & No & No & No \\
\hline $\begin{array}{l}\text { 'Han FN Q' } \\
\text { 'Han FN Q' }\end{array}$ & $\begin{array}{l}\text { R1 } \\
\text { E1 }\end{array}$ & $\begin{array}{l}11 / 28 / 2018 \\
11 / 29 / 2018\end{array}$ & $\begin{array}{l}0 / 0 \\
0 / 0\end{array}$ & $\begin{array}{l}1 / 0 \\
1 / 0\end{array}$ & $\begin{array}{l}0 / 0 \\
0 / 0\end{array}$ & $\begin{array}{l}0 / 0 \\
0 / 0\end{array}$ & $\begin{array}{l}2 / 0 \\
2 / 0\end{array}$ & $\begin{array}{l}2 / 1 \\
2 / 1\end{array}$ & $\begin{array}{l}\text { No } \\
\text { No }\end{array}$ & $\begin{array}{l}\text { No } \\
\text { No }\end{array}$ & $\begin{array}{l}\text { No } \\
\text { No }\end{array}$ & $\begin{array}{l}\text { No } \\
\text { No }\end{array}$ \\
\hline $\begin{array}{l}\text { 'Han FN Q' } \\
\text { 'Han FN Q' }\end{array}$ & $\begin{array}{l}\text { R2 } \\
\text { E2 }\end{array}$ & $\begin{array}{l}12 / 22 / 2018 \\
12 / 23 / 2018\end{array}$ & $\begin{array}{l}0 / 0 \\
0 / 0\end{array}$ & $\begin{array}{l}0 / 0 \\
0 / 0\end{array}$ & $\begin{array}{l}0 / 0 \\
0 / 0\end{array}$ & $\begin{array}{l}0 / 0 \\
0 / 0\end{array}$ & $\begin{array}{l}0 / 0 \\
0 / 0\end{array}$ & $\begin{array}{l}0 / 0 \\
0 / 0\end{array}$ & $\begin{array}{l}\text { No } \\
\text { No }\end{array}$ & $\begin{array}{l}\text { No } \\
\text { No }\end{array}$ & $\begin{array}{l}\text { No } \\
\text { No }\end{array}$ & $\begin{array}{l}\text { No } \\
\text { No }\end{array}$ \\
\hline 'Han FN Q' & R3 & $01 / 09 / 2019$ & - & $0 / 0$ & $0 / 0$ & $0 / 0$ & $0 / 0$ & $0 / 0$ & No & No & No & No \\
\hline
\end{tabular}

Table 12. Crop development stages—Flowering—Chinese cultivars—Southern biotypes (2018-2019)

\begin{tabular}{|c|c|c|c|c|c|c|c|c|c|c|c|c|}
\hline \multirow{2}{*}{\multicolumn{2}{|c|}{$\begin{array}{l}\text { Variety Field } \\
\text { Observation Date }\end{array}$}} & \multirow[t]{2}{*}{$\begin{array}{l}\text { Sowing } \\
\text { Date }\end{array}$} & \multicolumn{6}{|c|}{$\begin{array}{c}\begin{array}{c}\text { Flowering } \\
\text { (Male/Female) }\end{array} \\
(0=\text { no floral initiation; } 1 \text { =floral initiation; } 2=\text { inflorescence development; } 3=\text { full flowering })\end{array}$} & \multicolumn{4}{|c|}{$\begin{array}{l}\text { Seed Set } \\
(\text { Yes/No) }\end{array}$} \\
\hline & & & 01/02/2019 & 01/16/2019 & $01 / 22 / 2019$ & $01 / 29 / 2019$ & 02/06/2019 & 02/13/2019 & 01/22/2019 & 01/29/2019 & $02 / 06 / 2019$ & $02 / 13 / 2019$ \\
\hline $\begin{array}{l}\text { ‘Bama' } \\
\text { ‘Bama' }\end{array}$ & $\begin{array}{l}\text { R1 } \\
\text { E1 }\end{array}$ & $\begin{array}{l}11 / 28 / 2018 \\
11 / 29 / 2018\end{array}$ & $\begin{array}{l}0 / 0 \\
0 / 0\end{array}$ & $\begin{array}{l}0 / 0 \\
0 / 0\end{array}$ & $\begin{array}{l}0 / 0 \\
0 / 0\end{array}$ & $\begin{array}{l}1 / 1 \\
0 / 0\end{array}$ & $\begin{array}{l}1 / 1 \\
1 / 0\end{array}$ & $\begin{array}{l}2 / 1 \\
1 / 1\end{array}$ & $\begin{array}{l}\text { No } \\
\text { No }\end{array}$ & $\begin{array}{l}\text { No } \\
\text { No }\end{array}$ & $\begin{array}{l}\text { No } \\
\text { No }\end{array}$ & $\begin{array}{l}\text { No } \\
\text { No }\end{array}$ \\
\hline $\begin{array}{l}\text { 'Bama' } \\
\text { 'Bama' }\end{array}$ & $\begin{array}{l}\text { R2 } \\
\text { E2 }\end{array}$ & $\begin{array}{l}12 / 22 / 2018 \\
12 / 23 / 2018\end{array}$ & $\begin{array}{l}0 / 0 \\
0 / 0\end{array}$ & $\begin{array}{l}0 / 0 \\
0 / 0\end{array}$ & $\begin{array}{l}0 / 0 \\
0 / 0\end{array}$ & $\begin{array}{l}0 / 0 \\
0 / 0\end{array}$ & $\begin{array}{l}1 / 0 \\
1 / 0\end{array}$ & $\begin{array}{l}1 / 0 \\
1 / 0\end{array}$ & $\begin{array}{l}\text { No } \\
\text { No }\end{array}$ & $\begin{array}{l}\text { No } \\
\text { No }\end{array}$ & $\begin{array}{l}\text { No } \\
\text { No }\end{array}$ & $\begin{array}{l}\text { No } \\
\text { No }\end{array}$ \\
\hline 'Bama' & R3 & $01 / 09 / 2019$ & $0 / 0$ & $0 / 0$ & $0 / 0$ & $0 / 0$ & $0 / 0$ & $0 / 0$ & No & No & No & No \\
\hline $\begin{array}{l}\text { 'Puma 3' } \\
\text { 'Puma 3' }\end{array}$ & $\begin{array}{l}\text { R1 } \\
\text { E1 }\end{array}$ & $\begin{array}{l}11 / 28 / 2018 \\
11 / 29 / 2018\end{array}$ & $\begin{array}{l}0 / 0 \\
0 / 0\end{array}$ & $\begin{array}{l}0 / 0 \\
0 / 0\end{array}$ & $\begin{array}{l}0 / 0 \\
0 / 0\end{array}$ & $\begin{array}{l}1 / 0 \\
0 / 0\end{array}$ & $\begin{array}{l}1 / 0 \\
1 / 0\end{array}$ & $\begin{array}{l}2 / 1 \\
1 / 0\end{array}$ & $\begin{array}{l}\text { No } \\
\text { No }\end{array}$ & $\begin{array}{l}\text { No } \\
\text { No }\end{array}$ & $\begin{array}{l}\text { No } \\
\text { No }\end{array}$ & $\begin{array}{l}\text { No } \\
\text { No }\end{array}$ \\
\hline $\begin{array}{l}\text { 'Puma 3' } \\
\text { 'Puma 3' }\end{array}$ & $\begin{array}{l}\text { R2 } \\
\text { E2 }\end{array}$ & $\begin{array}{l}12 / 22 / 2018 \\
12 / 23 / 2018\end{array}$ & $\begin{array}{l}0 / 0 \\
0 / 0\end{array}$ & $\begin{array}{l}0 / 0 \\
0 / 0\end{array}$ & $\begin{array}{l}0 / 0 \\
0 / 0\end{array}$ & $\begin{array}{l}0 / 0 \\
0 / 0\end{array}$ & $\begin{array}{l}0 / 0 \\
0 / 0\end{array}$ & $\begin{array}{l}1 / 0 \\
1 / 0\end{array}$ & $\begin{array}{l}\text { No } \\
\text { No }\end{array}$ & $\begin{array}{l}\text { No } \\
\text { No }\end{array}$ & $\begin{array}{l}\text { No } \\
\text { No }\end{array}$ & $\begin{array}{l}\text { No } \\
\text { No }\end{array}$ \\
\hline 'Puma 3' & R3 & $01 / 09 / 2019$ & $0 / 0$ & $0 / 0$ & $0 / 0$ & $0 / 0$ & $0 / 0$ & $0 / 0$ & No & No & No & No \\
\hline $\begin{array}{l}\text { 'Yuma' } \\
\text { 'Yuma' }\end{array}$ & $\begin{array}{l}\text { R1 } \\
\text { E1 }\end{array}$ & $\begin{array}{l}11 / 28 / 2018 \\
11 / 29 / 2018\end{array}$ & $\begin{array}{l}0 / 0 \\
0 / 0\end{array}$ & $\begin{array}{l}0 / 0 \\
0 / 0\end{array}$ & $\begin{array}{l}0 / 0 \\
0 / 0\end{array}$ & $\begin{array}{l}1 / 0 \\
1 / 0\end{array}$ & $\begin{array}{l}1 / 1 \\
1 / 0\end{array}$ & $\begin{array}{l}2 / 1 \\
1 / 1\end{array}$ & $\begin{array}{l}\text { No } \\
\text { No }\end{array}$ & $\begin{array}{l}\text { No } \\
\text { No }\end{array}$ & $\begin{array}{l}\text { No } \\
\text { No }\end{array}$ & $\begin{array}{l}\text { No } \\
\text { No }\end{array}$ \\
\hline $\begin{array}{l}\text { 'Yuma' } \\
\text { 'Yuma' }\end{array}$ & $\begin{array}{l}\text { R2 } \\
\text { E2 }\end{array}$ & $\begin{array}{l}12 / 22 / 2018 \\
12 / 23 / 2018\end{array}$ & $\begin{array}{l}0 / 0 \\
0 / 0\end{array}$ & $\begin{array}{l}0 / 0 \\
0 / 0\end{array}$ & $\begin{array}{l}0 / 0 \\
0 / 0\end{array}$ & $\begin{array}{l}0 / 0 \\
0 / 0\end{array}$ & $\begin{array}{l}1 / 0 \\
1 / 0\end{array}$ & $\begin{array}{l}1 / 1 \\
1 / 0\end{array}$ & $\begin{array}{l}\text { No } \\
\text { No }\end{array}$ & $\begin{array}{l}\text { No } \\
\text { No }\end{array}$ & $\begin{array}{l}\text { No } \\
\text { No }\end{array}$ & $\begin{array}{l}\text { No } \\
\text { No }\end{array}$ \\
\hline 'Yuma' & R3 & $01 / 09 / 2019$ & $0 / 0$ & $0 / 0$ & $0 / 0$ & $0 / 0$ & $0 / 0$ & $0 / 0$ & No & No & No & No \\
\hline $\begin{array}{l}\text { 'Si-1' } \\
\text { 'Si-1' }\end{array}$ & $\begin{array}{l}\text { R1 } \\
\text { E1 }\end{array}$ & $\begin{array}{l}11 / 28 / 2018 \\
11 / 29 / 2018\end{array}$ & $\begin{array}{l}0 / 0 \\
0 / 0\end{array}$ & $\begin{array}{l}0 / 0 \\
0 / 0\end{array}$ & $\begin{array}{l}0 / 0 \\
0 / 0\end{array}$ & $\begin{array}{l}0 / 0 \\
0 / 0\end{array}$ & $\begin{array}{l}1 / 0 \\
0 / 0\end{array}$ & $\begin{array}{l}2 / 1 \\
0 / 0\end{array}$ & $\begin{array}{l}\text { No } \\
\text { No }\end{array}$ & $\begin{array}{l}\text { No } \\
\text { No }\end{array}$ & $\begin{array}{l}\text { No } \\
\text { No }\end{array}$ & $\begin{array}{l}\text { No } \\
\text { No }\end{array}$ \\
\hline $\begin{array}{l}\text { 'Si-1' } \\
\text { 'Si-1' }\end{array}$ & $\begin{array}{l}\mathrm{R} 2 \\
\mathrm{E} 2\end{array}$ & $\begin{array}{l}12 / 22 / 2018 \\
12 / 23 / 2018\end{array}$ & $\begin{array}{l}0 / 0 \\
0 / 0\end{array}$ & $\begin{array}{l}0 / 0 \\
0 / 0\end{array}$ & $\begin{array}{l}0 / 0 \\
0 / 0\end{array}$ & $\begin{array}{l}0 / 0 \\
0 / 0\end{array}$ & $\begin{array}{l}0 / 0 \\
0 / 0\end{array}$ & $\begin{array}{l}0 / 0 \\
0 / 0\end{array}$ & $\begin{array}{l}\text { No } \\
\text { No }\end{array}$ & $\begin{array}{l}\text { No } \\
\text { No }\end{array}$ & $\begin{array}{l}\text { No } \\
\text { No }\end{array}$ & $\begin{array}{l}\text { No } \\
\text { No }\end{array}$ \\
\hline 'Si-1' & R3 & $01 / 09 / 2019$ & $0 / 0$ & $0 / 0$ & $0 / 0$ & $0 / 0$ & $0 / 0$ & $0 / 0$ & No & No & No & No \\
\hline
\end{tabular}




\subsection{THC monitoring}

In accordance with local regulations THC levels were determined in the first sowings as each cultivar reached approximately fifty percent seed set (Table 13). Cultivars that set seed too late or not at all were not sampled.

Table 13. Cultivars, sampling dates and THC levels (2019)

\begin{tabular}{lll}
\hline Cultivar & $\begin{array}{l}\text { THC } \\
\text { (\% dry weight) }\end{array}$ & Pass/Fail \\
\hline 'CFX-1' & 0.1010 & Pass \\
'Katani' & 0.1570 & Pass \\
'Picolo' & 0.1000 & Pass \\
'Felina 32' & 0.0350 & Pass \\
'Ferimon' & 0.0320 & Pass \\
'Futura 75' & 0.0290 & Pass \\
'Santhica 70' & 0.0030 & Pass \\
'Han FN H' & Flowered too late & Untested \\
'Han FN Q' & Flowered too late & Untested \\
'Han Cold' & Flowered too late & Untested \\
'Han NE' & Flowered too late & Untested \\
'Han NW' & Flowered too late & Untested \\
'Bama' & 0.5060 & Fail \\
'Puma 3' & 0.2880 & Pass \\
'Yuma' & 0.7230 & Fail \\
'Si-1' & Flowered too late & Untested \\
\hline
\end{tabular}

\subsection{Seed yields}

All the Canadian and French cultivars produced seed which was harvested with a grain header in mid-April a month after it was ripe and later than ideal. An undetermined amount of seed was lost to wind, bird and mammal damage while awaiting harvest, and seed was lost during the harvest from the shattering of overly dry plants (Table 14).

Table 14. Seed yields-Canadian and French cultivars (2019)

\begin{tabular}{llllll}
\hline Cultivar & Site & $\begin{array}{c}\text { Seed Yield } \\
(\mathrm{kg})\end{array}$ & $\begin{array}{c}\text { Area } \\
\text { Harvested } \\
\text { (ha) }\end{array}$ & $\begin{array}{c}\text { Seed Yield } \\
(\mathrm{kg} / \mathrm{ha})\end{array}$ & $\begin{array}{c}\text { Seed Yield } \\
\text { (Average) } \\
(\mathrm{kg} / \mathrm{ha})\end{array}$ \\
\hline 'CFX-1' & E2 & 278 & 0.27 & 1030 & 1215 \\
& R2 & 1093 & 0.78 & 1400 & \\
‘CRS-1' & E2 & 669 & 0.63 & 1060 & 1085 \\
& R2 & 708 & 0.64 & 1110 & \\
'Katani' & E2 & 393 & 0.35 & 1120 & 1165 \\
& R2 & 534 & 0.44 & 1210 & \\
'Picolo' & E2 & 694 & 0.71 & 980 & 980 \\
& R2 & 521 & 0.53 & 980 & \\
'Felina 32' & E1+E2 & 244 & $0.13+0.16$ & 840 & 800 \\
& R1+R2 & 198 & $0.16+0.10$ & 760 & \\
'Ferimon' & E1+E2 & 226 & $0.15+0.13$ & 810 & 768 \\
& R1 & 116 & 0.16 & 725 & \\
'Futura 75' & E1+E2 & 178 & $0.13+0.14$ & 660 & 555 \\
& R1 & 59 & 0.13 & 450 & \\
'Santhica 70' & E1+E2 & 175 & $0.14+0.14$ & 625 & 475 \\
& R1 & 52 & 0.16 & 325 & \\
\hline
\end{tabular}

\section{Discussion}

Cultivars developed differently at the replicate sites. Generally, all cultivars developed more nodes earlier and stalks elongated more rapidly to reach greater average heights at site E than at site R. This is likely because site E has lighter sandier soils allowing more rapid root growth and earlier crop establishment than site R. Differences in crop development were most pronounced in the French and especially the Chinese cultivars while Canadian cultivars showed less difference in growth and development between sites. This is likely because the Canadian cultivars flowered first ceasing nodal development and upward growth before more inter-plant differences could appear. 
Irrigation was well managed at both sites and the trial plots never lacked sufficient water. Nutrients and herbicides were applied as recommended and weed control measures were mostly effective with weeds appearing sporadically only where canopy closure was slower and/or only partial. Seed harvest was later than expected but was carried out efficiently.

Seed sowing rates were effectively predicted based on seed viability and achieved the projected stand density resulting in canopy closure of all trial plots. Sowing dates achieved various results with different cultivars based on their time of flowering. The Canadian cultivars were only sown once (12/22-23/2018) and judging from premature flowering should have performed better if sown two weeks earlier. The French cultivars in the second sowing (12/22-23/2018) were only slightly shorter than those of the first sowing (11/28-29/2018) and likely would be shorter and easier to harvest if sown two weeks later than the second sowing. Only a few Chinese cultivars flowered and those that did not flower were still growing taller when the trials were terminated. The earlier the Chinese cultivars were sown the taller they grew.

Pest infestations were minimal and caused few problems. Moderate infestations of Heliothis caterpillars caused some seed loss by consuming seeds at the milk stage. The 2018-2019 season had fewer than normal Heliothis problems. No other pests accounted for any economic damage. Nightly visitations were by wallabies, kangaroos, wombats and snakes.

All of the Canadian (0.10-0.16 percent THC) and French (0.01-0.04 percent THC) cultivars matured sufficiently to be sampled for their THC content and all were found to be well below the 0.3 percent allowed threshold. The French cultivars were especially low in THC content. This is likely because they are monoecious which allowed the inbreeding of single low-THC individuals to establish parental breeding lines. Three of the southern Chinese cultivars were sampled and only 'Puma 3' ( 0.29 percent THC) tested slightly below the allowable threshold while both 'Bama' (0.51 percent THC) and 'Yuma' ( 0.72 percent THC) tested well above the threshold. The remaining Chinese cultivars flowered too late to require testing.

All of the Canadian and French cultivars yielded mature seeds while none of the Chinese cultivars matured seed and they were not harvested. Average yields of the Canadian cultivars ranged from 980 to 1,215 kg/ha which is economically favorable and in line with local expectations. The French cultivars yielded considerably less on average ranging from 475 to $800 \mathrm{~kg} / \mathrm{ha}$ which is below the economic threshold yield of more than 1,000 kg/ha. Low yields may be caused by inbreeding depression resulting from inbreeding monoecious individuals to achieve low THC content.

Recommended agricultural practices were followed and trial populations grew as expected. Variations between crop development at the two sites highlight the importance of field selection and the enhancement of growth in plots with sandier soil. Germination testing seed batches to determine their viability and vigor before sowing resulted in rapid and nearly complete canopy closure with effective weed suppression.

Weed suppression was enhanced by spraying herbicides at sowing and before canopy closure. However, there exists an economic trade-off between herbicide applications and other forms of weed suppression such as sowing at a higher rate to encourage early and dense canopy establishment. Self-thinning (death or stunting of the shaded shorter plants) during canopy development assures that the canopy develops fully and shades out competing weeds. Sowing more seeds creates a tighter canopy earlier in development while maximizing potential stalk or seed yields, but also adds to the farmer's costs. For example, sowing viable seed (90 percent germination) at $50 \mathrm{~kg} / \mathrm{ha}$ rather than $35 \mathrm{~kg} / \mathrm{ha}$ requires nearly 50 percent more seed, which at a cost of $\$ 10$ per kg or more for sowing seed adds at least $\$ 150$ per hectare to the farmer's expenses. Would the expense of over-seeding at $50 \mathrm{~kg} / \mathrm{ha}$ ( $\$ 150.00$ per hectare) be balanced by not using herbicides?

Four herbicide applications cost $\$ 140.00$ per hectare for boom spraying and pre-emergent herbicide costs were more than $\$ 50.00$ per hectare with a total cost of weed suppression reaching nearly $\$ 200.00$ per hectare. At both of the Bothwell trial plots herbicide application rates could have been higher to achieve more effective chemical control. If sowing crops at a higher rate achieves nearly the same canopy establishment then the savings will be about $\$ 50.00$ per hectare. In addition, organically grown hemp seed commands a premium price which should also add to the farmer's profits.

The dioecious Canadian cultivars performed well for grain seed production with the highest yields. Early flowering, seed maturation and harvest result in reduced field time and allow the Canadian cultivars to fit more easily into existing crop rotations. Short crop height also accommodates the restrictions imposed by mechanical harvesting. Both sowings of French cultivars yielded much less seed than the Canadian cultivars and grew to heights nearly beyond the reach of a standard grain header. If European hemp harvesters were used rather than grain headers the French cultivars could be economically grown for both seeds and stalk biomass. Both the northern and southern Chinese cultivars flowered too late to produce viable seeds within the time constraints of the Bothwell variety trials and are not suitable for hemp seed grain production in Tasmania, although their vigorous growth and late flowering makes them good candidates for biomass production. THC levels in the sampled Chinese cultivars were too high for seed production, but if they do not flower at Tasmanian latitudes then THC content becomes irrelevant, and harvest machinery, fiber extraction technology 
and biomass processing infrastructure become the limiting factors.

Tasmania is well suited for growing Canadian seed cultivars and should appropriate processing infrastructure become available could also become a significant producer of hemp fiber and stalk biomass.

\section{References}

[1] Jobling, T. and P. Warner. (2000). New Tropical Industrial Hemp. Australian Hemp Resource and Manufacture, Ashgrove, Queensland, Australia: p. 5.

[2] Boersma, Mark, Timothy Smallbon, Ann-Marie Donoghue, Omid Ansari, A. J. Gracie. (2018) Industrial hemp production trials in Tasmania. Australian Industrial Hemp Conference Proceedings, Geelong, 27 February-2 March, 2018: 91-97.

[3] Skewes, Mark, Omid Ansari, Klara Marosszeky, Blair Jepson, Colin Steddy. (2018) Industrial Hemp Production Trials in South Australia. Australian Industrial Hemp Conference Proceedings, Geelong, 27 February-2 March, 2018: 98-104.

[4] Boersma, Mark, Timothy Smallbon, Ann-Marie Donoghue, Omid Ansari, A. J. Gracie. (2018). Industrial hemp production trials in Tasmania. Australian Industrial Hemp Conference Proceedings, Geelong, 27 February-2 March, 2018: 91-97.

[5] Tasmanian Irrigation. (2012). Tasmanian Hemp Industry Inquiry: 2.

[6] Skewes, Mark, Omid Ansari, Klara Marosszeky, Blair Jepson, Colin Steddy. (2018). Industrial Hemp Production Trials in South Australia. Australian Industrial Hemp Conference Proceedings, Geelong, 27 February-2 March, 2018: 98-104. 